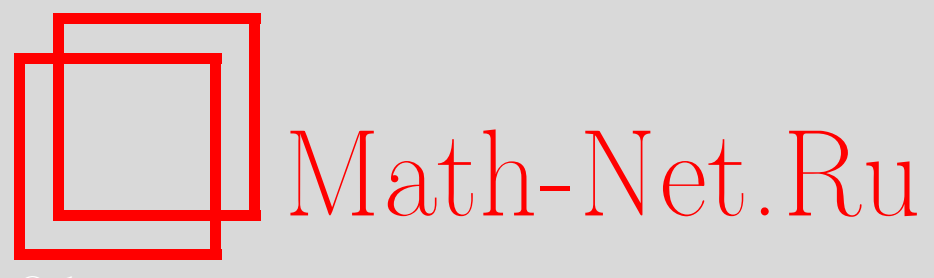

Т. А. Мельник, А. В. Попов, Асимптотический анализ краевых и спектральных задач в тонких перфорированных областях с быстро изменяющейся толщиной и различными предельными размерностями, Матем. сб., 2012, том 203, номер 8, 97-124

DOI: https://doi.org/10.4213/sm7862

Использование Общероссийского математического портала Math-Net.Ru подразумевает, что вы прочитали и согласны с пользовательским соглашением http: //www . mathnet.ru/rus/agreement

Параметры загрузки:

IP : 54.198 .55 .26

26 апреля 2023 г., 18:28:02

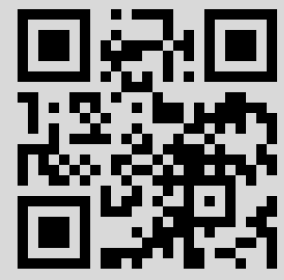




\author{
Т. А. Мельник, А. В. Попов
}

\title{
Асимптотический анализ краевых и спектральных задач в тонких перфорированных областях с быстро изменяющейся толщиной и различными предельными размерностями
}

\begin{abstract}
Рассматриваются краевые и спектральные задачи для эллиптического дифференциального уравнения с быстро осциллирующими коэффициентами в тонких перфорированных областях с быстро изменяющейся толщиной. В работе совмещается описание асимптотических алгоритмов для решений таких задач в тонких областях с различными предельными размерностями. Для смешанной неоднородной краевой задачи построен корректор и доказана асимптотическая оценка в соответствующем соболевском пространстве. Получены также асимптотические оценки для собственных значений и собственных функций спектральной задачи Неймана. При некоторых условиях симметрии на структуру тонкой перфорированной области и на коэффициенты уравнения построены полные асимптотические разложения для собственных значений и собственных функций.

Библиография: 21 название.
\end{abstract}

Ключевые слова: асимптотические приближения и разложения, тонкие перфорированные области, эллиптические краевые и спектральные задачи.

\section{§1. Введение}

В связи с быстрыми темпами развития микротехнологий, возобновился интерес к изучению краевых задач в тонких областях (один из линейных размеров такой области значительно меньше других).

Строгий асимптотический метод построения приближений в тонких областях был впервые предложен в работах А.Л. Гольденвейзера [1], [2]; дальнейшей его разработке посвящены работы М. Г. Джавалова [3], С. А. Назарова [4], [5], А. Б. Васильевой, В.Ф. Бутузова [6], D. Caillerie [7]. В этих работах в качестве тонких областей рассматривались области цилиндрического типа и основной подход для асимптотического исследования таких задач состоял в введении специальной замены переменных так, чтобы растянутая область не зависела от малого параметра. При этом малый параметр появлялся при старших производных в соответствующих дифференциальных уравнениях и далее применялся метод Люстерника-Вишика [8].

Эти методы не подходят для изучения краевых задач в тонких перфорированных областях с быстро изменяющейся толщиной. Методы теории усреднения для тонких областей впервые были использованы Г.П. Панасенко и М. В. Резцовым [9] при изучении трехмерной задачи теории упругости в неоднородной тонкой цилиндрической пластинке. В работе Т. А. Мельника [10] 
изучались эллиптические и спектральные задачи с быстро осциллирующими коэффициентами в тонких перфорированных областях с быстро изменяющейся толщиной. Исследованию асимптотического поведения решений разных краевых задач в тонких областях с быстро изменяющейся толщиной посвящены также работы [11]-[14].

В работе [15] С.А. Назаровым была выявлена тождественность процедур усреднения эллиптических краевых задач с быстро осциллирующими коэффициентами в тонких квазислоях цилиндрического типа в $\mathbb{R}^{d} \times \mathbb{R}^{n-d}$ и построения канонической системы жордановых цепочек полиномиальных эллиптических пучков. С помощью этого построения были исследованы основные характеристики усредненных операторов (размеры, порядки элементов, эллиптичность и формальная самосопряженность). Однако, как указано в самой работе [15; введение] этот метод не упрощает процедуру усреднения и при обосновании асимптотики необходимо вводить три группы ограничений, которые сужают сферу применимости этих результатов.

В монографии [16] подробно изложена асимптотическая теория тонких упругих пластин и стержней и изучаются только главные члены асимптотических решений, описаны новые методы исследований краевых и спектральных задач в тонких областях и приведена обширная библиография по этой тематике.

В настоящей работе независимо от предельной размерности тонкой области (например, тонкая пластина или стержень) совмещаются асимптотические алгоритмы для построения асимптотик решений краевых задач в тонких перфорированных областях с быстро изменяющейся толщиной и различными предельными размерностями. В $\S 2$ для решения смешанной неоднородной эллиптической краевой задачи с быстро осциллирующими коэффициентами построен корректор и доказана асимптотическая оценка в соответствующем соболевском пространстве. Модернизацией абстрактной схемы О. А. Олейник, Г. А. Иосифьяна и А. С. Шамаева [17; гл. III, §1] в §3 исследуется спектральная задача с однородными условиями Неймана на границах полостей. При некоторых условиях симметрии на структуру тонкой перфорированной области и на коэффициенты уравнения в $\ 4$ построены полные асимптотические разложения для собственных значений и собственных функций. В этом случае получены асимптотические оценки для скорости сходимости собственных значений порядка $O\left(\varepsilon^{2}\right)$ (см. следствие 4.1), где $\varepsilon$ - малый параметр, характеризующий относительную толщину тонкой области. Заметим, что аналогичные оценки в работах [10] и [16; гл. 7] имеют порядок $O(\sqrt{\varepsilon})$.

\section{1. Описание тонкой перфорированной области $c$ с быстро изме-} няющейся толщиной. Пусть функции $h_{ \pm}^{(1)}\left(\xi^{\prime}\right), h_{ \pm}^{(2)}\left(\xi^{\prime}\right), \ldots, h_{ \pm}^{(d)}\left(\xi^{\prime}\right)$ гладкие, положительные и 1 -периодические относительно переменных $\xi_{1}, \ldots, \xi_{n-d}$, где

$$
\xi^{\prime}:=\left(\xi_{1}, \ldots, \xi_{n-d}\right) \in \mathbb{R}^{n-d}, \quad d, n \in \mathbb{N}, \quad d<n .
$$

С помощью этих функций определим область

$$
\begin{gathered}
\omega=\left\{\xi:=\left(\xi_{1}, \ldots, \xi_{n}\right) \in \mathbb{R}^{n}: 0<\xi_{i}<1, i=1, \ldots, n-d,\right. \\
\left.-h_{-}^{(k)}\left(\xi^{\prime}\right)<\xi_{n-d+k}<h_{+}^{(k)}\left(\xi^{\prime}\right), k=1, \ldots, d\right\} .
\end{gathered}
$$


Пусть $T_{0}$ - конечное объединение замкнутых областей класса $C^{2, \alpha}$, которые принадлежат $\omega$ и не пересекаются между собой. Введем такие обозначения:

$$
\omega_{0}=\omega \backslash T_{0}, \quad T_{0}^{\varepsilon}=\varepsilon \cdot T_{0}=\left\{x: \varepsilon^{-1} x \in T_{0}\right\}, \quad T^{\varepsilon}=\bigcup_{\mathbf{z}_{0} \in \mathbb{Z}^{n}}\left(T_{0}^{\varepsilon}+\varepsilon \mathbf{z}_{0}\right),
$$

где $\mathbf{z}_{0}=\left(z_{1}, \ldots, z_{n-d}, 0, \ldots, 0\right), \varepsilon-$ малый положительный параметр.

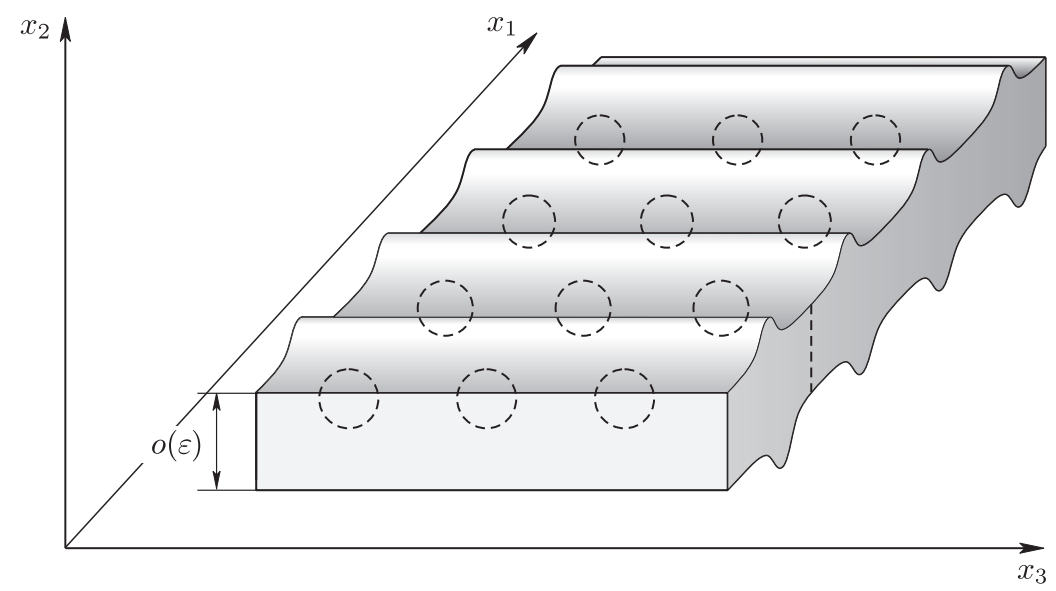

Рис. 1. Модельная тонкая область $\Omega_{\varepsilon}$ при $n=3, d=1$

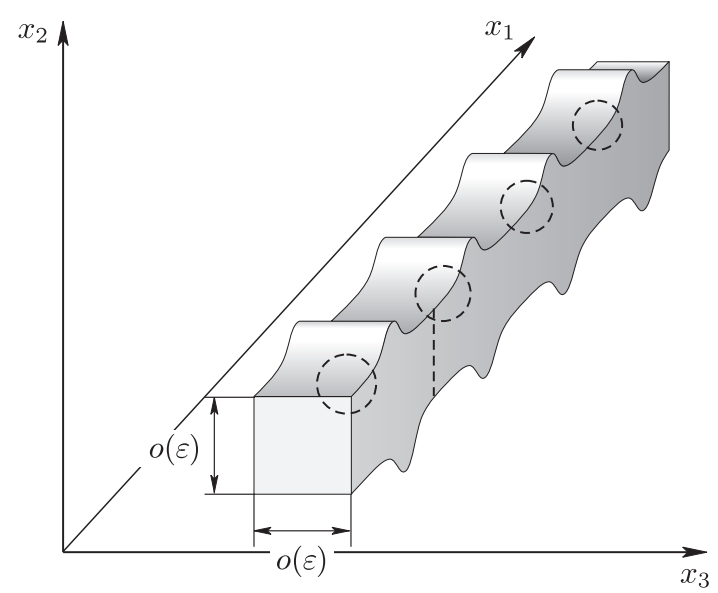

Рис. 2. Модельная тонкая область $\Omega_{\varepsilon}$ при $n=3, d=2$

Пусть $\Omega$ - ограниченная область в $\mathbb{R}^{n-d}, \partial \Omega \in C^{3}$. Модельная тонкая перфорированная область с предельной размерностью $n-d$ определяется следующим 
образом: $\Omega_{\varepsilon}=Q_{\varepsilon} \backslash T^{\varepsilon}$ (см. рис. 1,2$)$, где

$$
\begin{aligned}
Q_{\varepsilon}=\{x & =\left(x_{1}, \ldots, x_{n-d}, x_{n-d+1}, \ldots, x_{n}\right) \in \mathbb{R}^{n}: x^{\prime}=\left(x_{1}, \ldots, x_{n-d}\right) \in \Omega, \\
& \left.-\varepsilon h_{-}^{(k)}\left(\frac{x^{\prime}}{\varepsilon}\right)<x_{n-d+k}<\varepsilon h_{+}^{(k)}\left(\frac{x^{\prime}}{\varepsilon}\right), k=1, \ldots, d\right\} .
\end{aligned}
$$

Не ограничивая общности и для избежания в дальнейшем дополнительных технических вычислений будем считать, что $\partial T^{\varepsilon} \cap \partial \Omega=\varnothing$. Для частей границы области $\Omega_{\varepsilon}$ введем такие обозначения:

$$
\begin{aligned}
& S_{\varepsilon}^{ \pm, i}=\left\{x: x^{\prime} \in \Omega, x_{n-d+i}= \pm \varepsilon h_{ \pm}^{(i)}\left(\frac{x^{\prime}}{\varepsilon}\right),\right. \\
&\left.x_{n-d+k} \in\left(-\varepsilon h_{-}^{(k)}\left(\frac{x^{\prime}}{\varepsilon}\right), \varepsilon h_{+}^{(k)}\left(\frac{x^{\prime}}{\varepsilon}\right)\right), k=1, \ldots, d, i \neq k\right\}, \\
& S_{\varepsilon}^{ \pm}=\bigcup_{i=1}^{d} S_{\varepsilon}^{ \pm, i}, \quad G_{\varepsilon}=\partial T^{\varepsilon} \cap Q_{\varepsilon}, \quad \Gamma_{\varepsilon}=\partial \Omega_{\varepsilon} \backslash\left(S_{\varepsilon}^{ \pm} \cup Q_{\varepsilon}\right) .
\end{aligned}
$$

В дальнейшем также будем использовать тонкую область

$$
\widetilde{Q}_{\varepsilon}=\left\{x=\left(x^{\prime}, x^{\prime \prime}\right) \in \mathbb{R}^{n}: x^{\prime} \in \Omega, x^{\prime \prime} \in K_{\varepsilon}^{(d)}:=\left(-\varepsilon H_{-}, \varepsilon H_{+}\right)^{d}\right\},
$$

которая содержит $Q_{\varepsilon}$. Здесь $x^{\prime \prime}=\left(x_{n-d+1}, x_{n-d+2}, \ldots, x_{n}\right)$ и

$$
H_{ \pm}:=1+\max _{i=1, d} \max _{\xi^{\prime} \in \mathbb{R}^{n-d}}\left|h_{ \pm}^{(i)}\left(\xi^{\prime}\right)\right| \text {. }
$$

\section{§ 2. Усреднение и построение корректора для эллиптических краевых задач}

Пусть

$$
L_{\varepsilon} \equiv \sum_{i, j=1}^{n} \frac{\partial}{\partial x_{i}}\left(a_{i j}\left(\frac{x}{\varepsilon}\right) \frac{\partial}{\partial x_{j}}\right)
$$

- симметрический, равномерно эллиптический дифференциальный оператор, т.е.

$$
a_{i j}(\xi)=a_{j i}(\xi) \quad \forall \xi \in \mathbb{R}^{n} \quad \forall i, j=1, \ldots, n
$$

и существуют константы $\chi_{1}>0$ и $\chi_{2}>0$ такие, что

$$
\chi_{1}|\eta|^{2} \leqslant \sum_{i, j=1}^{n} a_{i j}(\xi) \eta_{i} \eta_{j} \leqslant \chi_{2}|\eta|^{2} \quad \forall \xi \in \mathbb{R}^{n} \quad \forall \eta \in \mathbb{R}^{n} .
$$

ЗАмЕчАниЕ 1. В дальнейшем мы будем опускать символ суммирования по индексам $i, j$ от 1 до $n$, которые повторяются в каком-либо выражении.

В области $\Omega_{\varepsilon}$ рассмотрим следующую задачу:

$$
\begin{cases}L_{\varepsilon}\left(u_{\varepsilon}\right)=f_{\varepsilon} & \text { в } \Omega_{\varepsilon}, \\ \sigma_{\varepsilon}\left(u_{\varepsilon}\right)=0 & \text { на } G_{\varepsilon}, \\ \sigma_{\varepsilon}\left(u_{\varepsilon}\right)=\varepsilon g_{\varepsilon}^{ \pm} & \text {на } S_{\varepsilon}^{ \pm}, \\ u_{\varepsilon}=\varphi_{0} & \text { на } \Gamma_{\varepsilon},\end{cases}
$$


где

$$
\sigma_{\varepsilon}\left(u_{\varepsilon}\right) \equiv a_{i j}^{\varepsilon} \partial_{x_{j}} u_{\varepsilon} \nu_{i}\left(\frac{x}{\varepsilon}\right)
$$

- производная по конормали,

$$
\left(\nu_{1}\left(\frac{x}{\varepsilon}\right), \ldots, \nu_{n}\left(\frac{x}{\varepsilon}\right)\right)
$$

- внешняя нормаль к $\partial \Omega_{\varepsilon}$,

$$
\partial_{x_{j}} u_{\varepsilon}:=\frac{\partial u_{\varepsilon}}{\partial x_{j}}, \quad a_{i j}^{\varepsilon}:=a_{i j}\left(\frac{x}{\varepsilon}\right), \quad f_{\varepsilon}:=f\left(x^{\prime}, \frac{x}{\varepsilon}\right), \quad g_{\varepsilon}^{ \pm}:=g^{ \pm}\left(x^{\prime}, \frac{x}{\varepsilon}\right) .
$$

Данные задачи удовлетворяют условиям: функции $a_{i j}(\xi), f\left(x^{\prime}, \xi\right), g^{ \pm}\left(x^{\prime}, \xi\right)$, $x^{\prime} \in \mathbb{R}^{n-d}, \xi \in \mathbb{R}^{n}, i, j=1, \ldots, n, 1$-периодические по переменным $\xi_{1}, \ldots, \xi_{n-d}$, $a_{i j} \in C^{1, \alpha}, f$ и $g^{ \pm}$имеют непрерывные производные первого порядка по $x^{\prime}$, как и сами функции, принадлежат соответственно $C^{0, \alpha}, C^{1, \alpha}$ по переменной $\xi$ равномерно относительно $x^{\prime}$ (т.е. постоянные в определении соответствующих пространств Гёльдера не зависят от $\left.x^{\prime}\right), \varphi_{0} \in H^{3}(\Omega)$.

ОПРЕДЕЛЕНИЕ 1. Обобщенным решением задачи (2.1) при фиксированном значении параметра $\varepsilon$ называется функция $u_{\varepsilon} \in H^{1}\left(\Omega_{\varepsilon}\right)$ такая, что $u_{\varepsilon}-\varphi_{0} \in$ $H^{1}\left(\Omega_{\varepsilon} ; \Gamma_{\varepsilon}\right)=\left\{v \in H^{1}\left(\Omega_{\varepsilon}\right):\left.v\right|_{\Gamma_{\varepsilon}}=0\right\}$ и

$$
\int_{\Omega_{\varepsilon}} a_{i j}^{\varepsilon} \partial_{x_{i}} u_{\varepsilon} \partial_{x_{j}} \psi d x=-\int_{\Omega_{\varepsilon}} f_{\varepsilon} \psi d x+\varepsilon \int_{S_{\varepsilon}^{ \pm}} g_{\varepsilon}^{ \pm} \psi d \sigma_{x} \quad \forall \psi \in H^{1}\left(\Omega_{\varepsilon} ; \Gamma_{\varepsilon}\right) .
$$

Изучим асимптотическое поведение решения $u_{\varepsilon}$ задачи $(2.1)$ при $\varepsilon \rightarrow 0$.

2.1. Вспомогательные неравенства. Так же, как и в [17; гл. I, §4.2], строим операторы продолжения

$$
\mathscr{P}_{0}: H^{1}\left(\omega_{0}\right) \mapsto H^{1}(\omega), \quad \mathscr{P}_{\varepsilon}: H^{1}\left(\Omega_{\varepsilon}\right) \mapsto H^{1}\left(Q_{\varepsilon}\right), \quad \widetilde{\mathscr{P}}_{\varepsilon}: H^{1}\left(\Omega_{\varepsilon}\right) \mapsto H^{1}\left(\widetilde{Q}_{\varepsilon}\right)
$$

такие, что $\mathscr{P}_{0} u=u, \mathscr{P}_{\varepsilon} u=u, \widetilde{P}_{\varepsilon} u=u$, если $u=$ const, $и$

$$
\begin{array}{cc}
\left\|\mathscr{P}_{0} u\right\|_{H^{1}(\omega)} \leqslant c_{1}\|u\|_{H^{1}\left(\omega_{0}\right)},\left\|\nabla_{\xi} \mathscr{P}_{0} u\right\|_{L^{2}(\omega)} \leqslant c_{1}\left\|\nabla_{\xi} u\right\|_{L^{2}\left(\omega_{0}\right)} & \forall u \in H^{1}\left(\omega_{0}\right), \\
\left\|\mathscr{P}_{\varepsilon} u\right\|_{H^{1}\left(Q_{\varepsilon}\right)} \leqslant c_{2}\|u\|_{H^{1}\left(\Omega_{\varepsilon}\right)},\left\|\nabla_{x} \mathscr{P}_{\varepsilon} u\right\|_{L^{2}\left(Q_{\varepsilon}\right)} \leqslant c_{2}\left\|\nabla_{x} u\right\|_{L^{2}\left(\Omega_{\varepsilon}\right)} & \forall u \in H^{1}\left(\Omega_{\varepsilon}\right), \\
\left\|\widetilde{P}_{\varepsilon} u\right\|_{H^{1}\left(\widetilde{Q}_{\varepsilon}\right)} \leqslant c_{3}\|u\|_{H^{1}\left(\Omega_{\varepsilon}\right)},\left\|\nabla_{x} \widetilde{\mathscr{P}}_{\varepsilon} u\right\|_{L^{2}\left(\widetilde{Q}_{\varepsilon}\right)} \leqslant c_{3}\left\|\nabla_{x} u\right\|_{L^{2}\left(\Omega_{\varepsilon}\right)} & \forall u \in H^{1}\left(\Omega_{\varepsilon}\right) .
\end{array}
$$

ЗАмечАнИЕ 2. Здесь и далее все постоянные $c_{1}, c_{2}, \ldots, C_{1}, C_{2}, \ldots$ не зависят от функции $u$ и параметра $\varepsilon$.

Покажем, что постоянная в неравенстве Фридрикса для функций из пространства $H^{1}\left(\Omega_{\varepsilon} ; \Gamma_{\varepsilon}\right)$ не зависит от $\varepsilon$. Пусть $u \in C^{\infty}\left(\bar{\Omega}_{\varepsilon}\right) \cap H^{1}\left(\Omega_{\varepsilon} ; \Gamma_{\varepsilon}\right)$. Поскольку $\|u\|_{L^{2}\left(\Omega_{\varepsilon}\right)} \leqslant\left\|\mathscr{P}_{\varepsilon} u\right\|_{L^{2}\left(Q_{\varepsilon}\right)}$, то обозначая $v=\mathscr{P}_{\varepsilon} u$ (очевидно, что $v_{\mid \partial \Omega}=0$ ) 
и разбивая область $Q_{\varepsilon}$ гиперплоскостями $\left\{x_{n-d+1}=0\right\}, \ldots,\left\{x_{n}=0\right\}$ на $2 d$ частей, получаем

$$
\begin{aligned}
& \int_{\Omega} \int_{0}^{\varepsilon h_{+}^{(1)}\left(x^{\prime} / \varepsilon\right)} \ldots \int_{0}^{\varepsilon h_{+}^{(d)}\left(x^{\prime} / \varepsilon\right)} v^{2}(x) d x^{\prime \prime} d x^{\prime}=\left|y_{n-d+i}=\frac{x_{n-d+i}}{h_{+}^{(i)}\left(x^{\prime} / \varepsilon\right)}, i=1, \ldots, d\right| \\
& \quad=\int_{[0, \varepsilon]^{d}} \int_{\Omega} v^{2}\left(x^{\prime}, y_{n-d+1} h_{+}^{(1)}, \ldots, y_{n} h_{+}^{(d)}\right) \prod_{i=1}^{d} h_{+}^{(i)}\left(\frac{x^{\prime}}{\varepsilon}\right) d x^{\prime} d y^{\prime \prime} \\
& \leqslant C_{1} \int_{[0, \varepsilon]^{d}} \int_{\Omega} \sum_{i=1}^{n-d}\left(\partial_{x_{i}} v+\varepsilon^{-1} \sum_{j=1}^{d} y_{n-d+j} \frac{\partial h_{+}^{(j)}}{\partial \xi_{i}} \partial_{x_{n-d+j}} v\right)^{2} d x^{\prime} d y^{\prime \prime} \\
& \leqslant C_{2} \int_{[0, \varepsilon]^{d}} \int_{\Omega}\left[\sum_{i=1}^{n-d}\left(\partial_{x_{i}} v\right)^{2}+\sum_{j=1}^{d}\left(\frac{y_{n-d+j}}{\varepsilon}\right)^{2}\left(\frac{\partial h_{+}^{(j)}}{\partial \xi_{i}}\right)^{2}\left(\partial_{x_{n-d+j}} v\right)^{2}\right] d x^{\prime} d y^{\prime \prime} .
\end{aligned}
$$

Так как $y_{n-d+j} \in(0, \varepsilon)$, то

$$
\begin{aligned}
& \int_{\Omega} \int_{0}^{\varepsilon h_{+}^{(1)}\left(x^{\prime} / \varepsilon\right)} \cdots \int_{0}^{\varepsilon h_{+}^{(d)}\left(x^{\prime} / \varepsilon\right)} v^{2}(x) d x^{\prime \prime} d x^{\prime} \\
& \leqslant C_{3} \int_{\Omega} \int_{0}^{\varepsilon h_{+}^{(1)}\left(x^{\prime} / \varepsilon\right)} \cdots \int_{0}^{\varepsilon h_{+}^{(d)}\left(x^{\prime} / \varepsilon\right)} \frac{\left|\nabla_{x} v(x)\right|^{2}}{\prod_{i=1}^{d} h_{+}^{(i)}\left(x^{\prime} / \varepsilon\right)} d x \\
& \leqslant C_{4} \int_{\Omega} \int_{0}^{\varepsilon h_{+}^{(1)}\left(x^{\prime} / \varepsilon\right)} \cdots \int_{0}^{\varepsilon h_{+}^{(d)}\left(x^{\prime} / \varepsilon\right)}\left|\nabla_{x} v\right|^{2} d x,
\end{aligned}
$$

где постоянная $C_{2}$ зависит только от области $\Omega$ и от максимума и минимума функций $\left\{h_{+}^{(i)}\right\}$, а также максимума $\left|\nabla_{\xi^{\prime}} h_{+}^{(i)}\left(\xi^{\prime}\right)\right|$. Таким образом, на основании неравенств $(2.3)$ имеем, что для всех функций $u \in H^{1}\left(\Omega_{\varepsilon} ; \Gamma_{\varepsilon}\right)$

$$
\|u\|_{L^{2}\left(\Omega_{\varepsilon}\right)} \leqslant C_{3}\left\|\nabla_{x} \mathscr{P}_{\varepsilon} u\right\|_{L^{2}\left(Q_{\varepsilon}\right)} \leqslant C_{4}\left\|\nabla_{x} u\right\|_{L^{2}\left(\Omega_{\varepsilon}\right)} .
$$

Оценим теперь норму следа функции $u$ на $S_{\varepsilon}^{ \pm}$. Очевидно, что след этой функции на $S_{\varepsilon}^{ \pm}$совпадает со следом ее продолжения $\mathscr{P}_{\varepsilon} u$ на область $Q_{\varepsilon}$. Тогда для любого $i \in\{1, \ldots, d\}$

$$
T_{\varepsilon}^{(i)} u:=\left.\mathscr{P}_{\varepsilon} u(x)\right|_{x_{n-d+i}=\varepsilon h_{+}^{(i)}\left(x^{\prime} / \varepsilon\right)}=\int_{x_{n-d+i}}^{\varepsilon h_{+}^{(i)}\left(x^{\prime} / \varepsilon\right)} \frac{\partial \mathscr{P}_{\varepsilon} u(x)}{\partial x_{n-d+i}} d x_{n-d+i}+\mathscr{P}_{\varepsilon} u(x),
$$

для всех $x_{n-d+i} \in\left(0, \varepsilon h_{+}^{(i)}\left(x^{\prime} / \varepsilon\right)\right)$. Возведем обе части этого неравенства в квадрат и применим неравенство Коши-Буняковского:

$$
\left(T_{\varepsilon}^{(i)} u\right)^{2} \leqslant 2\left(\varepsilon c_{1} \int_{0}^{\varepsilon h_{+}^{(i)}\left(x^{\prime} / \varepsilon\right)}\left(\frac{\partial \mathscr{P}_{\varepsilon} u(x)}{\partial x_{n-d+i}}\right)^{2} d x_{n-d+i}+\left(\mathscr{P}_{\varepsilon} u\right)^{2}(x)\right) .
$$

Домножив полученное равенство на соответствующий элемент поверхности $S_{\varepsilon}^{+, i}$ и проинтегрировав по

$$
\Omega \times \prod_{j=1, j \neq i}^{d}\left(-\varepsilon h_{-}^{(j)}\left(\frac{x^{\prime}}{\varepsilon}\right), \varepsilon h_{+}^{(j)}\left(\frac{x^{\prime}}{\varepsilon}\right)\right)
$$


получаем:

$$
\begin{aligned}
\left\|\mathscr{P}_{\varepsilon} u\right\|_{L^{2}\left(S^{ \pm, i}\right)}^{2} & \leqslant c_{2}\left(\varepsilon \int_{\Omega_{\varepsilon}}\left(\frac{\partial \mathscr{P}_{\varepsilon} u(x)}{\partial x_{n-d+i}}\right)^{2} d x+\varepsilon^{-1} \int_{\Omega_{\varepsilon}}\left(\mathscr{P}_{\varepsilon} u\right)^{2}(x) d x\right) \\
& \leqslant \frac{c_{3}}{\varepsilon}\left\|\mathscr{P}_{\varepsilon} u\right\|_{H^{1}\left(Q_{\varepsilon}\right)}^{2} .
\end{aligned}
$$

Аналогичные вычисления сделаем для поверхности $S_{\varepsilon}^{-, i}$. Таким образом, на основе свойств оператора продолжения $\mathscr{P}_{\varepsilon}$, выводим

$$
\|u\|_{L^{2}\left(S_{\varepsilon}^{ \pm}\right)}^{2}=\sum_{i=1}^{d}\|u\|_{L^{2}\left(S_{\varepsilon}^{ \pm, i}\right)}^{2} \leqslant c_{3} \varepsilon^{-1}\|u\|_{H^{1}\left(\Omega_{\varepsilon}\right)}^{2} \quad \forall u \in H^{1}\left(\Omega_{\varepsilon}\right) .
$$

Теперь оценим след функции $u$ на $G_{\varepsilon}$. Обозначим через $I_{\varepsilon}$ множество таких $\mathbf{z}_{0} \in \mathbb{Z}^{n}, z_{n-d+1}=0, \ldots, z_{n}=0$, что $Q_{\varepsilon} \cap\left(T_{0}^{\varepsilon}+\varepsilon \mathbf{z}_{0}\right) \neq \varnothing$. Принимая во внимание тот факт, что $\|\phi\|_{L^{2}\left(\partial T_{0}\right)} \leqslant c_{1}\|\phi\|_{H^{1}\left(\omega_{0}\right)}$ для всех $\phi \in H^{1}\left(\omega_{0}\right)$, имеем

$$
\begin{aligned}
\|u\|_{L^{2}\left(G_{\varepsilon}\right)}^{2} & =\varepsilon^{n-1} \sum_{\mathbf{z}_{0} \in I_{\varepsilon}} \int_{\partial T_{0}+\mathbf{z}_{0}} u^{2}(\varepsilon \xi) d \sigma_{\xi} \leqslant \varepsilon^{n-1} c_{2} \sum_{\mathbf{z}_{0} \in I_{\varepsilon}}\|u(\varepsilon \xi)\|_{H^{1}\left(\omega_{0}+\mathbf{z}_{0}\right)}^{2} \\
& \leqslant c_{2} \sum_{\mathbf{z}_{0} \in I_{\varepsilon}}\left(\varepsilon^{-1}\|u\|_{L^{2}\left(\varepsilon \omega_{0}+\varepsilon \mathbf{z}_{0}\right)}^{2}+\varepsilon\left\|\nabla_{x} u\right\|_{L^{2}\left(\varepsilon \omega_{0}+\varepsilon \mathbf{z}_{0}\right)}^{2}\right) \leqslant c_{3} \varepsilon^{-1}\|u\|_{H^{1}\left(\Omega_{\varepsilon}\right)}^{2} .
\end{aligned}
$$

Очевидно, что при некоторых $\mathbf{z}_{0} \in I_{\varepsilon}$ надо брать не всю область $\varepsilon \omega_{0}+\varepsilon \mathbf{z}_{0}$, а только некоторую ее часть, которая принадлежит $\Omega_{\varepsilon}$. Итак,

$$
\|u\|_{L^{2}\left(G_{\varepsilon}\right)} \leqslant c_{3} \varepsilon^{-1 / 2}\|u\|_{H^{1}\left(\Omega_{\varepsilon}\right)} \quad \forall u \in H^{1}\left(\Omega_{\varepsilon}\right) .
$$

Используя неравенства (2.5)-(2.7), стандартным методом доказываем существование и единственность обобщенного решения задачи

$$
\begin{cases}L_{\varepsilon}\left(u_{\varepsilon}\right)=f_{\varepsilon}+\partial_{x_{i}} f_{i}^{(\varepsilon)} & \text { в } \Omega_{\varepsilon}, \\ \sigma_{\varepsilon}\left(u_{\varepsilon}\right)=\varepsilon \psi_{0}+f_{i}^{(\varepsilon)} \nu_{i} & \text { на } G_{\varepsilon}, \\ \sigma_{\varepsilon}\left(u_{\varepsilon}\right)=\varepsilon g_{\varepsilon}^{ \pm}+f_{i}^{(\varepsilon)} \nu_{i} & \text { на } S_{\varepsilon}^{ \pm} \\ u_{\varepsilon}=\varphi_{0} & \text { на } \Gamma_{\varepsilon}\end{cases}
$$

где $f_{i}^{(\varepsilon)} \in L^{2}\left(\Omega_{\varepsilon}\right), \psi_{0} \in L^{2}\left(G_{\varepsilon}\right)$, а также априорную оценку

$$
\begin{aligned}
\left\|u_{\varepsilon}\right\|_{H^{1}\left(\Omega_{\varepsilon}\right)} \leqslant c_{1} & \left(\left\|f_{\varepsilon}\right\|_{L^{2}\left(\Omega_{\varepsilon}\right)}+\sum_{i=1}^{n}\left\|f_{i}^{\varepsilon}\right\|_{L^{2}\left(\Omega_{\varepsilon}\right)}\right. \\
& \left.+\sqrt{\varepsilon}\left(\left\|g_{\varepsilon}^{ \pm}\right\|_{L^{2}\left(S_{\varepsilon}^{ \pm}\right)}+\left\|\psi_{0}\right\|_{L^{2}\left(G_{\varepsilon}\right)}\right)+\left\|\varphi_{0}\right\|_{H^{1}(\Omega)}\right) .
\end{aligned}
$$

Очевидно, что соответствующая оценка имеет место для решения задачи (2.1).

2.2. Усредненная задача. Рассмотрим следующую задачу: найти функцию $N \in H_{\sharp}^{1}\left(\omega_{0}\right)$ такую, что

$$
\begin{cases}L_{\xi \xi} N(\xi)=F_{0}(\xi)+\partial_{\xi_{i}} F_{i}(\xi), & \xi \in \omega_{0}, \\ \sigma_{\xi}(N(\xi))=\Phi_{0}^{ \pm}(\xi)+F_{i}(\xi) \nu_{i}(\xi), & \xi \in S^{ \pm}, \\ \sigma_{\xi}(N(\xi))=\Phi_{1}(\xi)+F_{i}(\xi) \nu_{i}(\xi), & \xi \in \partial T_{0}, \\ \langle N\rangle_{\omega_{0}}=0 . & \end{cases}
$$


Здесь $H_{\sharp}^{1}\left(\omega_{0}\right):=\left\{v \in H^{1}\left(\omega_{0}\right): v-1\right.$-периодические по переменным $\left.\xi_{1}, \ldots, \xi_{n-d}\right\}$,

$$
\begin{gathered}
L_{\xi \xi}(N):=\partial_{\xi_{i}}\left(a_{i j}(\xi) \partial_{\xi_{j}} N\right), \quad \sigma_{\xi}(N):=a_{i j}(\xi) \partial_{\xi_{j}} N \nu_{i}(\xi), \\
\langle N\rangle_{\omega_{0}}=\frac{1}{\left|\omega_{0}\right|} \int_{\omega_{0}} N(\xi) d \xi
\end{gathered}
$$

$\left(\nu_{1}(\xi), \ldots, \nu_{n}(\xi)\right)$ - внешняя нормаль к $\partial \omega_{0}, \partial_{\xi_{i}} F=\frac{\partial F}{\partial \xi_{i}},\left|\omega_{0}\right|-$ мера Лебега области $\omega_{0},\left\{F_{0}, F_{1}, \ldots, F_{n}\right\} \subset L^{2}\left(\omega_{0}\right), \Phi_{1} \in L^{2}\left(\partial T_{0}\right), \Phi_{0}^{ \pm} \in L^{2}\left(S^{ \pm}\right)$,

$$
\begin{gathered}
S^{ \pm}=\bigcup_{i=1}^{d} S^{ \pm, i}, \\
S^{ \pm, i}=\left\{\xi: \xi^{\prime} \in[0,1]^{n-d}, \xi_{n-d+i}= \pm h_{ \pm}^{(i)}\left(\xi^{\prime}\right),\right. \\
\left.\xi_{n-d+k} \in\left(-\varepsilon h_{-}^{(k)}\left(\xi^{\prime}\right), \varepsilon h_{+}^{(k)}\left(\xi^{\prime}\right)\right), k=1, \ldots, d, k \neq i\right\} .
\end{gathered}
$$

ОПредЕлЕНИЕ 2. Функция $N \in H_{\sharp}^{1}\left(\omega_{0}\right)$ называется обобщенным решением задачи (2.10), если для любой функции $\psi \in H_{\sharp}^{1}\left(\omega_{0}\right)$

$$
\int_{\omega_{0}} a_{i j} \partial_{\xi_{j}} N \partial_{\xi_{i}} \psi d \xi=\int_{\omega_{0}}\left(F_{i} \partial_{\xi_{i}} \psi-F_{0} \psi\right) d \xi+\int_{\partial T_{0}} \Phi_{1} \psi d \sigma_{\xi}+\int_{S^{ \pm}} \Phi_{0}^{ \pm} \psi d \sigma_{\xi}
$$

Согласно теореме 1 из [18; дополнение], задача (2.10) имеет единственное обобщенное решение тогда и только тогда, когда

$$
\int_{\omega_{0}} F_{0}(\xi) d \xi=\int_{S^{+}} \Phi_{0}^{+}(\xi) d \sigma_{\xi}+\int_{S^{-}} \Phi_{0}^{-}(\xi) d \sigma_{\xi}+\int_{\partial T_{0}} \Phi_{1}(\xi) d \sigma_{\xi}
$$

Кроме того, имеет место оценка

$$
\|N\|_{H^{1}\left(\omega_{0}\right)} \leqslant C\left(\sum_{i=0}^{n}\left\|F_{i}\right\|_{L^{2}\left(\omega_{0}\right)}+\left\|\Phi_{1}\right\|_{L^{2}\left(\partial T_{0}\right)}+\left\|\Phi_{0}^{ \pm}\right\|_{L^{2}\left(S^{ \pm}\right)}\right)
$$

где постоянная $C$ не зависит от $N,\left\{F_{i}\right\}, \Phi_{0}^{ \pm}, \Phi_{1}$.

Для каждого $p=1, \ldots, n-d$ определим обобщенное решение $N_{p} \in H_{\sharp}^{1}\left(\omega_{0}\right)$ задачи

$$
\begin{cases}L_{\xi \xi}\left(N_{p}(\xi)\right)=-\partial_{\xi_{i}} a_{i p}(\xi), & \xi \in \omega_{0}, \\ \sigma_{\xi}\left(N_{p}(\xi)\right)=-a_{i p}(\xi) \nu_{i}(\xi), & \xi \in S^{ \pm} \cup \partial T_{0}, \\ \left\langle N_{p}\right\rangle_{\omega_{0}}=0 . & \end{cases}
$$

Очевидно, что условие разрешимости (2.12) для таких задач выполняется.

При помощи функций $\left\{N_{p}\right\}$ определим дифференциальный оператор

$$
\widehat{L}:=\sum_{p, q=1}^{n-d} \frac{\partial}{\partial x_{q}}\left(\widehat{a}_{p q} \frac{\partial}{\partial x_{p}}\right), \quad \widehat{a}_{p q}=\left\langle a_{p q}+\sum_{j=1}^{n} a_{p j} \frac{\partial N_{q}}{\partial \xi_{j}}\right\rangle_{\omega_{0}}, \quad p, q=1, \ldots, n-d .
$$

Аналогично, как и в доказательстве теоремы 1 из [18; гл. 4, §1], проверяем, что коэффициенты этого оператора удовлетворяют условиям симметрии и эллиптичности. Дифференциальный оператор $\widehat{L}$ называется усредненным дифференциальным оператором для оператора $L_{\varepsilon}$. 
Рассмотрим задачу

$$
\begin{cases}\widehat{L}\left(u_{0}\left(x^{\prime}\right)\right)=\widehat{F}\left(x^{\prime}\right), & x^{\prime} \in \Omega, \\ u_{0}\left(x^{\prime}\right)=\varphi_{0}\left(x^{\prime}\right), & x^{\prime} \in \partial \Omega,\end{cases}
$$

где

$$
\widehat{F}\left(x^{\prime}\right)=\left\langle f\left(x^{\prime}, \cdot\right)\right\rangle_{\omega_{0}}-\frac{1}{\left|\omega_{0}\right|}\left(\int_{S^{+}} g^{+}\left(x^{\prime}, \xi\right) d \sigma_{\xi}+\int_{S^{-}} g^{-}\left(x^{\prime}, \xi\right) d \sigma_{\xi}\right), \quad x^{\prime} \in \Omega .
$$

Лемма 2.1. Для любой функиии $u \in H^{1}\left(\Omega_{\varepsilon} ; \Gamma_{\varepsilon}\right)$ при достаточно малых значениях параметра є имеет место неравенство

$\left|\int_{\Omega_{\varepsilon}} f_{\varepsilon} u d x-\varepsilon\left(\int_{S_{\varepsilon}^{+}} g_{\varepsilon}^{+} u d \sigma_{x}+\int_{S_{\varepsilon}^{-}} g_{\varepsilon}^{-} u d \sigma_{x}\right)-\int_{\Omega_{\varepsilon}} \widehat{F} u d x\right| \leqslant c_{3} \varepsilon^{1+d / 2}\|u\|_{H^{1}\left(\Omega_{\varepsilon} ; \Gamma_{\varepsilon}\right)}$.

ДокАЗАТЕЛЬСтво. Согласно определению функции $\widehat{F}$ для каждого $x^{\prime} \in \Omega$ выполняется условие разрешимости $(2.12)$ для задачи: найти $N\left(x^{\prime}, \cdot\right) \in H_{\sharp}^{1}\left(\omega_{0}\right)$ такую, что

$$
\begin{cases}L_{\xi \xi}\left(N\left(x^{\prime}, \xi\right)\right)=f\left(x^{\prime}, \xi\right)-\widehat{F}\left(x^{\prime}\right), & \xi \in \omega_{0}, \\ \sigma_{\xi}\left(N\left(x^{\prime}, \xi\right)\right)=0, & \xi \in \partial T_{0}, \\ \sigma_{\xi}\left(N\left(x^{\prime}, \xi\right)\right)=g^{ \pm}\left(x^{\prime}, \xi\right), & \xi \in S^{ \pm}, \\ \left\langle N\left(x^{\prime}, \cdot\right)\right\rangle_{\omega_{0}}=0 . & \end{cases}
$$

В силу условий, наложенных на гладкость границы областей $T_{0}$ и на гладкость функций $h_{ \pm}, f, g_{ \pm}$, на основании теоремы 6.30 и неравенства (6.77) из [19] имеем

$$
\max _{x^{\prime} \in \Omega}\left\|N\left(x^{\prime}, \cdot\right)\right\|_{C^{2, \alpha}} \leqslant c_{1} \max _{x^{\prime} \in \Omega}\left(\left\|f\left(x^{\prime}, \cdot\right)\right\|_{C^{0, \alpha}}+\left\|g^{ \pm}\left(x^{\prime}, \cdot\right)\right\|_{C^{1, \alpha}}\right),
$$

откуда следует, что

$$
\int_{\Omega_{\varepsilon}}\left(\sum_{i=1}^{n} \frac{\partial N}{\partial \xi_{i}}\left(x^{\prime}, \frac{x}{\varepsilon}\right)\right)^{2} d x \leqslant c_{2} \varepsilon^{d} .
$$

Перепишем соотношения задачи $(2.16)$ в переменных $x$ :

$$
\begin{cases}\varepsilon \frac{\partial}{\partial x_{i}}\left(a_{i j}\left(\frac{x}{\varepsilon}\right) \frac{\partial N}{\partial \xi_{j}}\left(x^{\prime}, \frac{x}{\varepsilon}\right)\right)=f\left(x^{\prime}, \frac{x}{\varepsilon}\right)-\widehat{F}\left(x^{\prime}\right), & x \in \Omega_{\varepsilon}, \\ a_{i j}\left(\frac{x}{\varepsilon}\right) \frac{\partial N}{\partial \xi_{i}}\left(x^{\prime}, \frac{x}{\varepsilon}\right) \nu_{i}=\varepsilon g^{ \pm}\left(x^{\prime}, \frac{x}{\varepsilon}\right), & x \in S_{\varepsilon}^{ \pm}, \\ a_{i j}\left(\frac{x}{\varepsilon}\right) \frac{\partial N}{\partial \xi_{i}}\left(x^{\prime}, \frac{x}{\varepsilon}\right) \nu_{i}=0, & x \in G_{\varepsilon} .\end{cases}
$$

Умножая первое уравнение в $(2.18)$ на произвольную функцию $u \in H^{1}\left(\Omega_{\varepsilon} ; \Gamma_{\varepsilon}\right)$ и интегрируя по частям, получим

$$
\begin{gathered}
\varepsilon \int_{\Omega_{\varepsilon}} a_{i j}\left(\frac{x}{\varepsilon}\right) \frac{\partial N}{\partial \xi_{i}}\left(x^{\prime}, \frac{x}{\varepsilon}\right) \frac{\partial u}{\partial x_{i}} d x=\int_{\Omega_{\varepsilon}}\left(\widehat{F}\left(x^{\prime}\right)-f\left(x^{\prime}, \frac{x}{\varepsilon}\right)\right) u d x \\
+\varepsilon\left(\int_{S_{\varepsilon}^{+}} g^{+}\left(x^{\prime}, \frac{x}{\varepsilon}\right) u d \sigma_{x}+\int_{S_{\varepsilon}^{-}} g^{-}\left(x^{\prime}, \frac{x}{\varepsilon}\right) u d \sigma_{x}\right),
\end{gathered}
$$

откуда, с учетом $(2.17)$ и ограниченности $\left\{a_{i j}\right\}$, получаем утверждение леммы. 
Таким образом, правая часть задачи (2.15) близка в смысле леммы 2.1 к правым частям задачи (2.1). Задачу (2.15) будем называть усредненной задачей для задачи (2.1).

2.3. Асимптотическое приближение для решения задачи (2.1). В качестве приближенного решения задачи (2.1) возьмем функцию

$$
\widetilde{U}_{\varepsilon}(x):=u_{0}\left(x^{\prime}\right)+\varepsilon U_{1}\left(x^{\prime}, \frac{x}{\varepsilon}\right), \quad x \in \Omega_{\varepsilon},
$$

где $u_{0}$ - решение задачи $(2.15), U_{1}\left(x^{\prime}, \xi\right):=\sum_{p=1}^{n-d} N_{p}(\xi) \partial_{x_{p}} u_{0}\left(x^{\prime}\right), N_{p}$ - решение задачи (2.13).

Введем также обозначения

$$
L_{x \xi}=\frac{\partial}{\partial x_{i}} a_{i j}(\xi) \frac{\partial}{\partial \xi_{j}}+\frac{\partial}{\partial \xi_{i}} a_{i j}(\xi) \frac{\partial}{\partial x_{j}}, \quad L_{x x}=a_{i j}(\xi) \frac{\partial^{2}}{\partial x_{i} \partial x_{j}} .
$$

Легко проверить, что для любой функции $\phi \in C^{2}\left(\Omega \times \mathbb{R}_{\xi}^{n}\right)$ имеет место тождество

$$
L_{\varepsilon}\left(\phi\left(x^{\prime}, \frac{x}{\varepsilon}\right)\right)=\left.\left\{\left(\varepsilon^{-2} L_{\xi \xi}+\varepsilon^{-1} L_{x \xi}+L_{x x}\right)\left(\phi\left(x^{\prime}, \xi\right)\right)\right\}\right|_{\xi=x / \varepsilon} .
$$

Используя (2.20) и учитывая дифференциальные уравнения задач (2.13), выводим, что

$$
\begin{aligned}
L_{\varepsilon}\left(u_{\varepsilon}-\widetilde{U}_{\varepsilon}\right)= & f\left(x^{\prime}, \xi\right)-\varepsilon^{-1}\left(L_{\xi \xi} U_{1}\left(x^{\prime}, \xi\right)+L_{x \xi} u_{0}\left(x^{\prime}\right)\right) \\
& \quad-\left(L_{x \xi} U_{1}\left(x^{\prime}, \xi\right)+L_{x x} u_{0}\left(x^{\prime}\right)\right)-\varepsilon L_{x x} U_{1}\left(x^{\prime}, \xi\right) \\
= & f\left(x^{\prime}, \xi\right)-\left(L_{x \xi} U_{1}\left(x^{\prime}, \xi\right)+L_{x x} u_{0}\left(x^{\prime}\right)\right)-\varepsilon L_{x x} U_{1}\left(x^{\prime}, \xi\right), \quad \xi=\frac{x}{\varepsilon} .
\end{aligned}
$$

Определим 1-периодические по переменным $\xi^{\prime}$ функции $N_{p q}, p, q=1, \ldots$, $n-d$, как обобщенные решения краевых задач

$$
\begin{gathered}
L_{\xi \xi}\left(N_{p q}(\xi)\right)+\partial_{\xi_{i}}\left(a_{i q}(\xi) N_{p}\right)+a_{i q}(\xi) \partial_{\xi_{i}} N_{p}+a_{p q}(\xi)=\widehat{a}_{p q}, \quad \xi \in \omega_{0}, \\
\sigma_{\xi}\left(N_{p q}(\xi)\right)+a_{i q}(\xi) N_{p}(\xi) \nu_{i}(\xi)=0, \quad \xi \in S^{ \pm} \cup \partial T_{0}, \\
\left\langle N_{p q}\right\rangle_{\omega_{0}}=0 .
\end{gathered}
$$

Существование и единственность решений задач (2.22) следует из условия разрешимости (2.12). Используя эти решения, из (2.21) получаем

$$
L_{\varepsilon}\left(u_{\varepsilon}-\widetilde{U}_{\varepsilon}\right)=f\left(x^{\prime}, \frac{x}{\varepsilon}\right)-\widehat{F}\left(x^{\prime}\right)-\varepsilon \Psi_{0}-\varepsilon \sum_{k=1}^{n} \frac{\partial}{\partial x_{k}} \Psi_{k},
$$

где

$$
\begin{gathered}
\Psi_{0}=\sum_{k, p, q=1}^{n-d}\left(a_{k q} N_{p} \frac{\partial^{3} u_{0}}{\partial x_{k} \partial x_{q} \partial x_{p}}+\sum_{j=1}^{n} a_{k j} \frac{\partial N_{p q}}{\partial \xi_{j}} \frac{\partial^{3} u_{0}}{\partial x_{k} \partial x_{q} \partial x_{p}}\right), \\
\Psi_{k}=\sum_{p, q=1}^{n-d} \sum_{j=1}^{n} a_{k j} \frac{\partial N_{p q}}{\partial \xi_{j}} \frac{\partial^{2} u_{0}}{\partial x_{q} \partial x_{p}} .
\end{gathered}
$$


Краевые условия для разницы $u_{\varepsilon}-\widetilde{U}_{\varepsilon}$ имеют вид

$$
\begin{aligned}
\sigma_{\varepsilon}\left(u_{\varepsilon}-\widetilde{U}_{\varepsilon}\right) & =\varepsilon\left(g^{ \pm}\left(x^{\prime}, \frac{x}{\varepsilon}\right)+\Psi_{k} \nu_{k}\right), & & x \in S_{\varepsilon}^{ \pm}, \\
\sigma_{\varepsilon}\left(u_{\varepsilon}-\widetilde{U}_{\varepsilon}\right) & =\varepsilon \Psi_{k} \nu_{k}, & & x \in G_{\varepsilon}, \\
u_{\varepsilon}-\widetilde{U}_{\varepsilon} & =-\varepsilon U_{1}, & & x \in \Gamma_{\varepsilon} .
\end{aligned}
$$

Вследствие гладкости коэффициентов задачи, а также свойств функций $N_{p}$, $N_{p q}$ имеем, что

$$
\sum_{k=0}^{n}\left\|\Psi_{k}\right\|_{L^{2}\left(\Omega_{\varepsilon}\right)} \leqslant c_{1} \varepsilon^{d / 2}\left\|u_{0}\right\|_{H^{3}(\Omega)}
$$

Покажем, что $\left\|\varepsilon U_{1}\right\|_{H^{1 / 2}\left(\Gamma_{\varepsilon}\right)} \leqslant c_{2} \varepsilon^{d / 2+1 / 2}\left\|u_{0}\right\|_{H^{2}(\Omega)}$. Учитывая определение нормы $\|\cdot\|_{H^{1 / 2}\left(\Gamma_{\varepsilon}\right)}$, достаточно показать существование функции $\Psi_{\varepsilon} \in H^{1}\left(\Omega_{\varepsilon}\right)$ такой, что

$$
\Psi_{\varepsilon}+\varepsilon \sum_{p=1}^{n-d} N_{p} \partial_{x_{p}} u_{0} \in H^{1}\left(\Omega_{\varepsilon} ; \Gamma_{\varepsilon}\right), \quad\left\|\Psi_{\varepsilon}\right\|_{H^{1}\left(\Omega_{\varepsilon}\right)} \leqslant c_{2} \varepsilon^{d / 2+1 / 2}\left\|u_{0}\right\|_{H^{2}(\Omega)} .
$$

Выберем функцию $\varphi_{\varepsilon}$ из пространства $C^{\infty}\left(\bar{Q}_{\varepsilon}\right)$ такую, что

$$
\begin{gathered}
\varphi_{\varepsilon}(x)=1 \quad \text { при } \rho\left(x, \partial Q_{\varepsilon}\right) \leqslant \varepsilon, \quad \varphi_{\varepsilon}(x)=0 \quad \text { при } \rho\left(x, \partial Q_{\varepsilon}\right) \geqslant 2 \varepsilon, \\
\sup _{x \in Q_{\varepsilon}}\left|\nabla \varphi_{\varepsilon}\right| \leqslant C \varepsilon^{-1} .
\end{gathered}
$$

Положим

$$
\Psi_{\varepsilon}:=-\varepsilon \varphi_{\varepsilon} \sum_{p=1}^{n-d} N_{p} \partial_{x_{p}} u_{0}, \quad K_{\varepsilon}:=\left\{x: \rho\left(x, \partial Q_{\varepsilon}\right) \leqslant 2 \varepsilon\right\} \cap \Omega_{\varepsilon} .
$$

Тогда

$$
\Psi_{\varepsilon} \in H^{1}\left(\Omega_{\varepsilon}\right), \quad\left\|\Psi_{\varepsilon}\right\|_{H^{1}\left(\Omega_{\varepsilon}\right)} \leqslant C_{2}\left(\left\|u_{0}\right\|_{H^{1}\left(K_{\varepsilon}\right)}+\varepsilon\left\|u_{0}\right\|_{H^{2}\left(K_{\varepsilon}\right)}\right) .
$$

На основании леммы 1.5 из [17; гл. I] имеем $\left\|u_{0}\right\|_{H^{1}\left(K_{\varepsilon}\right)} \leqslant C_{3} \varepsilon^{1 / 2}\left\|u_{0}\right\|_{H^{2}\left(K_{\varepsilon}\right)}$. Отсюда получим $\left\|\Psi_{\varepsilon}\right\|_{H^{1}\left(\Omega_{\varepsilon}\right)} \leqslant C_{4} \varepsilon^{d / 2+1 / 2}\left\|u_{0}\right\|_{H^{2}(\Omega)}$. Поэтому в силу $(2.9)$ и леммы 2.1 из $(2.23)$ и (2.24) выводим

$$
\begin{aligned}
\left\|u_{\varepsilon}-\widetilde{U}_{\varepsilon}\right\|_{H^{1}\left(\Omega_{\varepsilon}\right)} & \leqslant C_{5}\left(\varepsilon^{d / 2+1}\left\|u_{0}\right\|_{H^{1}(\Omega)}+\varepsilon^{d / 2+1 / 2}\left\|u_{0}\right\|_{H^{3}(\Omega)}+\varepsilon^{d / 2+1 / 2}\left\|u_{0}\right\|_{H^{2}(\Omega)}\right) \\
& \leqslant C_{6} \varepsilon^{d / 2+1 / 2}\left(\|\widehat{F}\|_{H^{1}(\Omega)}+\left\|\varphi_{0}\right\|_{H^{5 / 2}(\partial \Omega)}\right) .
\end{aligned}
$$

Таким образом, доказана следующая

Теорема 1. Существуют положителъные константы с, $\varepsilon_{0}$ такие, что для всех значений $\varepsilon \in\left(0, \varepsilon_{0}\right)$

$$
\left\|u_{\varepsilon}-\widetilde{U}_{\varepsilon}\right\|_{H^{1}\left(\Omega_{\varepsilon}\right)} \leqslant c \varepsilon^{d / 2+1 / 2}
$$

где $u_{\varepsilon}$ - решение задачи (2.1), а функция $\widetilde{U}_{\varepsilon}$ определена в (2.19). 
СлеДСтвиЕ 2.1. Если $\Omega_{\varepsilon}=Q_{\varepsilon}$ (в этом случае $T_{0}=\varnothing, G_{\varepsilon}=\varnothing$, а в задачах (2.1), (2.13), (2.16) и (2.24) отсутствуют соответствующие краевые условия), mo

$$
\left\|E_{\varepsilon}\left(u_{\varepsilon}\right)-u_{0}\right\|_{L^{2}(\Omega)} \leqslant c_{1} \sqrt{\varepsilon}
$$

где

$$
\begin{gathered}
E_{\varepsilon}(u)\left(x^{\prime}\right)=\frac{1}{\left|\Pi_{\varepsilon}^{(d)}\right|} \int_{\Pi_{\varepsilon}^{(d)}} u\left(x^{\prime}, x^{\prime \prime}\right) d x^{\prime \prime} \\
\Pi_{\varepsilon}^{(d)}=\left(-\varepsilon h_{-}^{(1)}\left(\frac{x^{\prime}}{\varepsilon}\right), \varepsilon h_{+}^{(1)}\left(\frac{x^{\prime}}{\varepsilon}\right)\right) \times \cdots \times\left(-\varepsilon h_{-}^{(d)}\left(\frac{x^{\prime}}{\varepsilon}\right), \varepsilon h_{+}^{(d)}\left(\frac{x^{\prime}}{\varepsilon}\right)\right), \\
\left|\Pi_{\varepsilon}^{(d)}\right|=\int_{\Pi_{\varepsilon}^{(d)}} 1 d x^{\prime \prime} .
\end{gathered}
$$

ДокАзАтельство. Используя неравенство Коши-Буняковского и (2.25), выводим

$$
\begin{aligned}
& \left\|E_{\varepsilon}\left(u_{\varepsilon}\right)-u_{0}\right\|_{L^{2}(\Omega)}^{2}=\int_{\Omega}\left[\frac{1}{\left|\Pi_{\varepsilon}^{(d)}\right|} \int_{\Pi_{\varepsilon}^{(d)}} u_{\varepsilon}(x) d x^{\prime \prime}-u_{0}\left(x^{\prime}\right)\right]^{2} d x^{\prime} \\
& =\int_{\Omega}\left[\frac{1}{\left|\Pi_{\varepsilon}^{(d)}\right|} \int_{\Pi_{\varepsilon}^{(d)}} u_{\varepsilon}(x) d x^{\prime \prime}-\frac{1}{\left|\Pi_{\varepsilon}^{(d)}\right|} \int_{\Pi_{\varepsilon}^{(d)}} u_{0}\left(x^{\prime}\right) d x^{\prime \prime}\right]^{2} d x^{\prime} \\
& \leqslant c \varepsilon^{-2 d} \int_{\Omega}\left(\int_{\Pi_{\varepsilon}^{(d)}}\left(u_{\varepsilon}-u_{0}\right) d x^{\prime \prime}\right)^{2} d x^{\prime} \\
& \leqslant c_{1} \varepsilon^{-d}\left\|u_{\varepsilon}-u_{0}\right\|_{L^{2}\left(\Omega_{\varepsilon}\right)}^{2} \leqslant c_{2} \varepsilon^{-d} \varepsilon^{d+1}=c_{2} \varepsilon .
\end{aligned}
$$

Дальше мы будем использовать следующую лемму, которая доказывается аналогично неравенству $\left(6.7^{\prime}\right)$ из [20; гл. 1].

Лемма 2.2. Имеет место следующее неравенство:

$$
\left|\int_{\Omega}\left(E_{\varepsilon}\left(u^{2}\right)-E_{\varepsilon}^{2}(u)\right) d x^{\prime}\right| \leqslant c \varepsilon^{2-d}\|u\|_{H^{1}\left(\Omega_{\varepsilon}\right)}^{2} \quad \forall u \in H^{1}\left(\Omega_{\varepsilon}\right) .
$$

\section{§ 3. Асимптотические оценки}

\section{для решений спектральной задачи Неймана}

Теперь в области $\Omega_{\varepsilon}$ рассмотрим спектральную задачу

$$
\begin{cases}-L_{\varepsilon}\left(u^{\varepsilon}\right)=\lambda(\varepsilon) \rho\left(\frac{x}{\varepsilon}\right) u^{\varepsilon} & \text { в } \Omega_{\varepsilon}, \\ \sigma_{\varepsilon}\left(u^{\varepsilon}\right)=0 & \text { на } S_{\varepsilon}^{ \pm} \cup G_{\varepsilon}, \\ u^{\varepsilon}=0 & \text { на } \Gamma_{\varepsilon},\end{cases}
$$

где $\lambda(\varepsilon)$ - спектральный параметр, функция $\rho(\xi), \xi \in \mathbb{R}^{n}$, принадлежит $C^{0, \alpha}$, является 1-периодической относительно переменных $\xi^{\prime}$ и ограниченной положительными константами: $0<\rho_{0} \leqslant \rho \leqslant \rho_{1}$. Обозначим $\rho_{\varepsilon}(x):=\rho(x / \varepsilon), x \in \mathbb{R}^{n}$. 
Обобщенная постановка этой задачи состоит в том, чтобы найти функцию $u^{\varepsilon} \in H^{1}\left(\Omega_{\varepsilon} ; \Gamma_{\varepsilon}\right) \backslash\{0\}$ и число $\lambda(\varepsilon)$, для которых выполнено интегральное тождество

$$
\int_{\Omega_{\varepsilon}} a_{i j}^{\varepsilon} \partial_{x_{i}} u^{\varepsilon} \partial_{x_{j}} v d x=\lambda(\varepsilon) \int_{\Omega_{\varepsilon}} \rho_{\varepsilon} u^{\varepsilon} v d x \quad \forall v \in H^{1}\left(\Omega_{\varepsilon} ; \Gamma_{\varepsilon}\right) .
$$

Очевидно, что для каждого фиксированного $\varepsilon>0$ собственные значения задачи (3.1) формируют неубывающую последовательность

$$
0<c_{0} \leqslant \lambda_{1}(\varepsilon) \leqslant \cdots \leqslant \lambda_{n}(\varepsilon) \leqslant \cdots \rightarrow+\infty \quad \text { при } \quad n \rightarrow \infty,
$$

где каждое собственное значение считается столько раз, какова его кратность. Последовательность соответствующих собственных функций нормируем следующим образом:

$$
\int_{\Omega_{\varepsilon}} \rho_{\varepsilon}(x) u_{n}^{\varepsilon}(x) u_{m}^{\varepsilon}(x) d x=\varepsilon^{d} \delta_{n, m}, \quad n, m \in \mathbb{N},
$$

где $\delta_{n, m}-$ символ Кронекера.

Учитывая результаты предыдущего пункта и используя введенные там обозначения, выпишем усредненную спектральную задачу

$$
\widehat{L}(v)+\mu \widehat{\rho} v=0 \quad \text { в } \Omega, \quad v=0 \quad \text { на } \partial \Omega,
$$

где $\widehat{\rho}=\langle\rho\rangle_{\omega_{0}}=\left|\omega_{0}\right|^{-1} \int_{\omega_{0}} \rho(\xi) d \xi, \mu-$ спектральный параметр. Спектр задачи (3.5) состоит из неубывающей последовательности собственных значений конечной кратности:

$$
0<\mu_{1}<\mu_{2} \leqslant \cdots \leqslant \mu_{n} \leqslant \cdots \rightarrow+\infty \quad \text { при } \quad n \rightarrow \infty .
$$

Изучим асимптотическое поведение собственных значений и собственных функций задачи (3.1) при $\varepsilon \rightarrow 0$. Для этого используем абстрактную схему из работы [17; гл. III, §1], с помощью которой обосновывается асимптотическое поведение собственных значений и собственных функций последовательности операторов, заданных в различных гильбертовых пространствах. Однако, для спектральных задач в тонких областях некоторые условия этой схемы должны быть уточнены (впервые это было замечено в [10]).

Пусть $\mathscr{H}_{\varepsilon}, \mathscr{H}_{0}$ - сепарабельные гильбертовы пространства со скалярными произведениями $(u, v)_{\mathscr{H}_{\varepsilon}},(\varphi, \psi)_{\mathscr{H}_{0}}$ соответственно, и пусть $A_{\varepsilon}: \mathscr{H}_{\varepsilon} \mapsto \mathscr{H}_{\varepsilon}$, $A_{0}: \mathscr{H}_{0} \mapsto \mathscr{H}_{0}-$ линейные непрерывные операторы, причем $\operatorname{Im} A_{0} \subset \mathscr{V} \subset \mathscr{H}_{0}$, $\operatorname{Im} A_{0}:=\left\{v: v=A_{0} u, u \in \mathscr{H}_{0}\right\}, \mathscr{V}$ - линейное подпространство в $\mathscr{H}_{0}$.

В $[17 ;$ гл. III, $\S 1]$ для операторов $A_{\varepsilon}, A_{0}$ и пространств $\mathscr{H}_{\varepsilon}, \mathscr{H}_{0}$ предполагается выполнение следующих условий.

C1. Существуют линейные непрерывные операторы $R_{\varepsilon}: \mathscr{H}_{0} \rightarrow \mathscr{H}_{\varepsilon}$ такие, что для любого $f^{0} \in \mathscr{V}$

$$
\left(R_{\varepsilon} f^{0}, R_{\varepsilon} f^{0}\right)_{\mathscr{H}_{\varepsilon}} \rightarrow \gamma\left(f^{0}, f^{0}\right)_{\mathscr{H}_{0}} \quad \text { при } \quad \varepsilon \rightarrow 0,
$$

где $\gamma=$ const $>0$ не зависит от $f^{0}$. 
C2. Операторы $A_{\varepsilon}: \mathscr{H}_{\varepsilon} \mapsto \mathscr{H}_{\varepsilon}, A_{0}: \mathscr{H}_{0} \mapsto \mathscr{H}_{0}$ являются положительными, компактными и самосопряженными, причем их нормы ограничены постоянной, не зависящей от $\varepsilon$.

С3. Для любой $f \in \mathscr{V}$

$$
\left\|A_{\varepsilon} R_{\varepsilon} f-R_{\varepsilon} A_{0} f\right\|_{\mathscr{H}_{\varepsilon}} \rightarrow 0 \quad \text { при } \quad \varepsilon \rightarrow 0 .
$$

C4. Семейство операторов $\left\{A_{\varepsilon}\right\}$ равномерно компактно в следующем смысле. Из любой последовательности $f^{\varepsilon} \in \mathscr{H}_{\varepsilon}$ такой, что $\sup _{\varepsilon}\left\|f^{\varepsilon}\right\| \mathscr{H}_{\varepsilon}<\infty$ можно выбрать подпоследовательность $f^{\varepsilon^{\prime}}$ и найти вектор $w^{0} \in \mathscr{V}$ такие, что

$$
\left\|A_{\varepsilon^{\prime}} f^{\varepsilon^{\prime}}-R_{\varepsilon^{\prime}} w^{0}\right\|_{\mathscr{H}_{\varepsilon^{\prime}}} \rightarrow 0 \quad \text { при } \quad \varepsilon^{\prime} \rightarrow 0 .
$$

Применим эту схему к спектральным задачам (3.1) и (3.5). Через $\mathscr{H}_{\varepsilon}, \mathscr{H}_{0}$ обозначим пространства функций $L^{2}\left(\Omega_{\varepsilon}\right), L^{2}(\Omega)$, в которых скалярные произведения определены соответственно равенствами

$$
(u, v)_{\mathscr{H}_{\varepsilon}}=\frac{1}{\varepsilon^{d}} \int_{\Omega_{\varepsilon}} \rho_{\varepsilon} u v d x, \quad(\varphi, \psi)_{\mathscr{H}_{0}}=\widehat{\rho} \int_{\Omega} \varphi \psi d x^{\prime} .
$$

Операторы $A_{\varepsilon}: \mathscr{H}_{\varepsilon} \mapsto \mathscr{H}_{\varepsilon}$ и $A_{0}: \mathscr{H}_{0} \mapsto \mathscr{H}_{0}$ определим формулами $A_{\varepsilon} f^{\varepsilon}=u^{\varepsilon}$, $A_{0} f^{0}=u^{0}$, где $u^{\varepsilon}, u^{0}$ - обобщенные решения задач

$$
\begin{aligned}
& \begin{cases}-L_{\varepsilon}\left(u^{\varepsilon}\right)=\rho\left(\frac{x}{\varepsilon}\right) f^{\varepsilon} & \text { в } \Omega_{\varepsilon}, \\
\sigma_{\varepsilon}\left(u^{\varepsilon}\right)=0 & \text { на } S_{\varepsilon}^{ \pm} \cup G_{\varepsilon}, \\
u^{\varepsilon}=0 & \text { на } \Gamma_{\varepsilon},\end{cases} \\
& -\widehat{L}\left(u^{0}\right)=\widehat{\rho} f^{0} \quad \text { в } \Omega, \quad u^{0}=0 \quad \text { на } \partial \Omega
\end{aligned}
$$

соответственно. Положим $\mathscr{V}=\mathscr{H}_{0}$.

Проверим для операторов $A_{0}, A_{\varepsilon}$ условия C1-C4.

В качестве оператора $R_{\varepsilon}: \mathscr{H}_{0} \rightarrow \mathscr{H}_{\varepsilon}$ в условии $\mathrm{C} 1$ возьмем оператор, который каждой функции $v \in \mathscr{H}_{0}$ (ее можно рассматривать как функцию, заданную в $\left.L^{2}\left(Q_{\varepsilon}\right)\right)$ ставит в соответствие ее сужение на $\Omega_{\varepsilon}$, домноженное на $\left(\sqrt{\left|\omega_{0}\right|}\right)^{-1}$. Доопределим функцию $\rho_{\varepsilon}$ нулем на $Q_{\varepsilon} \backslash \Omega_{\varepsilon}$. Тогда согласно следствию 1.7 из [17; гл. I] будем иметь

$$
\begin{aligned}
& \frac{1}{\varepsilon^{d}} \int_{\Omega_{\varepsilon}} \rho_{\varepsilon}\left(R_{\varepsilon} v\right)^{2} d x=\int_{\Omega} v^{2}\left(x^{\prime}\right) \frac{1}{\left|\omega_{0}\right|} \int_{h_{-}^{(1)}\left(x^{\prime} / \varepsilon\right)}^{h_{+}^{(1)}\left(x^{\prime} / \varepsilon\right)} \cdots \int_{h_{-}^{(d)}\left(x^{\prime} / \varepsilon\right)}^{h_{+}^{(d)}\left(x^{\prime} / \varepsilon\right)} \rho\left(\frac{x^{\prime}}{\varepsilon}, \xi^{\prime \prime}\right) d \xi^{\prime \prime} d x^{\prime} \\
& \rightarrow \hat{\rho} \int_{\Omega} v^{2}\left(x^{\prime}\right) d x^{\prime} \quad \text { при } \varepsilon \rightarrow 0,
\end{aligned}
$$

а это означает выполнение условия С1. Кроме того, легко видеть, что нормы $\left\{\left\|R_{\varepsilon}\right\|\right\}_{\varepsilon>0}$ ограничены постоянной, не зависящей от $\varepsilon$.

Очевидно, что определенные выше операторы $A_{\varepsilon}, A_{0}$ будут самосопряженными и положительными. Ввиду компактности вложения $H^{1} \subset L^{2}$ они будут компактными. Согласно $(2.9)$ нормы $\left\{\left\|A_{\varepsilon}\right\|\right\}_{\varepsilon>0}$ ограничены постоянной, которая не зависит от параметра $\varepsilon$. Следовательно, имеет место условие С2. 
Пусть $f^{0} \in C^{\infty}(\bar{\Omega})$. Определим функции $u^{\varepsilon}=A_{\varepsilon} R_{\varepsilon} f^{0}, u^{0}=A_{0} f^{0}$. Вследствие неравенства $(2.9)\left\|u_{\varepsilon}\right\|_{H^{1}\left(\Omega_{\varepsilon} ; \Gamma_{\varepsilon}\right)} \leqslant c_{1} \sqrt{\varepsilon^{d}}$. Тогда $\left\|u_{\varepsilon}\right\|_{\mathscr{H}_{\varepsilon}} \leqslant c_{2}$. Соответствующая функция $\widehat{F}^{0}$, которая определяется в задаче $(2.15)$, равна $\widehat{F}^{0}=$ $\left(\sqrt{\left|\omega_{0}\right|}\right)^{-1} f^{0} \widehat{\rho}$. Поэтому на основании теоремы 1 имеет место неравенство

$$
\left\|u^{\varepsilon}-\left(\sqrt{\left|\omega_{0}\right|}\right)^{-1} u^{0}\right\|_{L^{2}\left(\Omega_{\varepsilon}\right)} \leqslant c_{3} \varepsilon^{d / 2+1 / 2}
$$

которое можно переписать в виде

$$
\left\|A_{\varepsilon} R_{\varepsilon} f^{0}-R_{\varepsilon} A_{0} f^{0}\right\|_{\mathscr{H}_{\varepsilon}} \leqslant c_{2} \varepsilon^{1 / 2} .
$$

Поскольку $C^{\infty}(\bar{\Omega})$ плотно в $\mathscr{H}_{0}$ и последовательности $\left\{\left\|A_{\varepsilon}\right\|\right\}_{\varepsilon>0}$ и $\left\{\left\|R_{\varepsilon}\right\|\right\}_{\varepsilon>0}$ ограничены, то это означает выполнение условия С3.

Условие С4 требует некоторой модификации, поскольку размерности задач (3.1) и (3.5) разные. Сначала заметим, что имеет место следующее утверждение.

Лемма 3.1. Существует линейный оператор $\mathrm{P}_{\varepsilon}: H^{1}\left(\Omega_{\varepsilon} ; \Gamma_{\varepsilon}\right) \mapsto H_{0}^{1}(\Omega)$ maкой, что для всякой функции $и \in H^{1}\left(\Omega_{\varepsilon} ; \Gamma_{\varepsilon}\right)$

$$
\left\|\mathrm{P}_{\varepsilon} u\right\|_{H^{1}(\Omega)} \leqslant c_{3} \varepsilon^{-d / 2}\|u\|_{H^{1}\left(\Omega_{\varepsilon}\right)} .
$$

ДокАЗАтельство. Оператор $\mathrm{P}_{\varepsilon}$ есть суперпозиция оператора $\widetilde{\mathscr{P}}_{\varepsilon}$ (см. (2.4)) и оператора

$$
\widetilde{E}_{\varepsilon}(u)=\left|K_{\varepsilon}^{(d)}\right|^{-1} \int_{K_{\varepsilon}^{(d)}} u\left(x^{\prime}, x^{\prime \prime}\right) d x^{\prime \prime}, \quad \text { где } \quad\left|K_{\varepsilon}^{(d)}\right|=\int_{K_{\varepsilon}^{(d)}} 1 d x^{\prime \prime},
$$

т.е. $\mathrm{P}_{\varepsilon}=\widetilde{E}_{\varepsilon} \circ \widetilde{P}_{\varepsilon} ;$ множество $K_{\varepsilon}^{(d)}$ определено в конце п. 1.1. Действительно, используя неравенство Коши-Буняковского и (2.4), имеем

$$
\begin{aligned}
& \left\|\widetilde{E}_{\varepsilon} \widetilde{\mathscr{P}}_{\varepsilon} u\right\|_{H^{1}(\Omega)}^{2} \\
& \quad=\int_{\Omega}\left[\left(\left|K_{\varepsilon}^{(d)}\right|^{-1} \int_{K_{\varepsilon}^{(d)}} \widetilde{\mathscr{P}}_{\varepsilon} u d x^{\prime \prime}\right)^{2}+\sum_{p=1}^{n-d}\left(\left|K_{\varepsilon}^{(d)}\right|^{-1} \int_{K_{\varepsilon}^{(d)}} \partial_{x_{p}}\left(\widetilde{\mathscr{P}}_{\varepsilon} u\right) d x^{\prime \prime}\right)^{2}\right]^{2} d x^{\prime} \\
& \quad \leqslant C_{1} \varepsilon^{-d}\left\|\widetilde{\mathscr{P}}_{\varepsilon} u\right\|_{H^{1}\left(\widetilde{Q}_{\varepsilon}\right)}^{2} \leqslant C_{2} \varepsilon^{-d}\|u\|_{H^{1}\left(\Omega_{\varepsilon}\right)}^{2} .
\end{aligned}
$$

Оператор $\mathrm{P}_{\varepsilon}$ обладает следующим свойством.

СледСТВиЕ 3.1. Пусть для последовательности $\left\{u_{\varepsilon}\right\}_{\varepsilon>0} \subset H^{1}\left(\Omega_{\varepsilon} ; \Gamma_{\varepsilon}\right)$ въполняется неравенство

$$
\sup _{\varepsilon>0}\left\|u_{\varepsilon}\right\|_{H^{1}\left(\Omega_{\varepsilon} ; \Gamma_{\varepsilon}\right)} \leqslant c_{4} \varepsilon^{d / 2} .
$$

Тогда

$$
\left\|u_{\varepsilon}-\mathrm{P}_{\varepsilon} u_{\varepsilon}\right\|_{\mathscr{H}_{\varepsilon}} \rightarrow 0 \quad \text { npu } \quad \varepsilon \rightarrow 0
$$


ДокАЗАТЕльство. Обозначая $U_{\varepsilon}:=\widetilde{\mathscr{P}}_{\varepsilon} u_{\varepsilon}$ и используя $(2.4)$ и лемму 2.2 , получаем

$$
\begin{aligned}
\| u_{\varepsilon} & -\mathrm{P}_{\varepsilon} u_{\varepsilon} \|_{\mathscr{H}_{\varepsilon}}^{2}=\frac{1}{\varepsilon^{d}} \int_{\Omega_{\varepsilon}} \rho_{\varepsilon}\left(u_{\varepsilon}-\mathrm{P}_{\varepsilon} u_{\varepsilon}\right)^{2} d x \leqslant \frac{c_{5}}{\varepsilon^{d}} \int_{\widetilde{Q}_{\varepsilon}}\left[\widetilde{\mathscr{P}}_{\varepsilon} u_{\varepsilon}-\widetilde{E}_{\varepsilon}\left(\widetilde{\mathscr{P}}_{\varepsilon} u_{\varepsilon}\right)\right]^{2} d x \\
& =\frac{c_{5}}{\varepsilon^{d}} \int_{\Omega} \int_{\left[-\varepsilon H_{-}, \varepsilon H_{+}\right]^{d}}\left[U_{\varepsilon}-\widetilde{E}_{\varepsilon}\left(U_{\varepsilon}\right)\right]^{2} d x^{\prime} d x^{\prime \prime} \\
& =\frac{c_{5}}{\varepsilon^{d}} \int_{\Omega} \int_{\left[-\varepsilon H_{-}, \varepsilon H_{+}\right]^{d}}\left[U_{\varepsilon}^{2}-2 U_{\varepsilon} \widetilde{E}_{\varepsilon}\left(U_{\varepsilon}\right)+\left(\widetilde{E}_{\varepsilon}\left(U_{\varepsilon}\right)\right)^{2}\right] d x^{\prime} d x^{\prime \prime} \\
& =c_{5}\left(H_{+}+H_{-}\right)^{d} \int_{\Omega}\left[\widetilde{E}_{\varepsilon}\left(U_{\varepsilon}^{2}\right)-\left(\widetilde{E}_{\varepsilon}\left(U_{\varepsilon}\right)\right)^{2}\right] d x^{\prime} \leqslant c_{5} \varepsilon^{2-d}\left\|\widetilde{\mathscr{P}}_{\varepsilon} u_{\varepsilon}\right\|_{H^{1}\left(\widetilde{Q}_{\varepsilon}\right)}^{2} \\
& \leqslant c_{6} \varepsilon^{2-d}\left\|u_{\varepsilon}\right\|_{H^{1}\left(\Omega_{\varepsilon}\right)}^{2} \leqslant c_{7} \varepsilon^{2} \rightarrow 0 \quad \text { при } \quad \varepsilon \rightarrow 0 .
\end{aligned}
$$

Пусть $\sup _{\varepsilon>0}\left\|f^{\varepsilon}\right\|_{\mathscr{H}_{\varepsilon}} \leqslant c_{4}$. Положим $u_{\varepsilon}=A_{\varepsilon} f^{\varepsilon}$. Тогда согласно неравенству $(2.9)\left\|A_{\varepsilon} f^{\varepsilon}\right\|_{H^{1}\left(\Omega_{\varepsilon}\right)} \leqslant c_{5} \sqrt{\varepsilon^{d}}$, а значит $\left\|\mathrm{P}_{\varepsilon} A_{\varepsilon} f^{\varepsilon}\right\|_{H^{1}(\Omega)} \leqslant c_{6}$. Благодаря компактности вложения $H^{1}(\Omega) \subset L^{2}(\Omega)$ существует функция $v_{0} \in H_{0}^{1}(\Omega)$ такая, что $\left\|\mathrm{P}_{\varepsilon^{\prime}} A_{\varepsilon^{\prime}} f^{\varepsilon^{\prime}}-v_{0}\right\|_{L^{2}(\Omega)} \rightarrow 0$ по некоторой подпоследовательности $\varepsilon^{\prime} \rightarrow 0$. Теперь, поскольку нормы операторов $R_{\varepsilon}$ равномерно ограничены относительно $\varepsilon$, то

$$
\left\|R_{\varepsilon^{\prime}} \mathrm{P}_{\varepsilon^{\prime}} A_{\varepsilon^{\prime}} f^{\varepsilon^{\prime}}-R_{\varepsilon^{\prime}} v_{0}\right\|_{\mathscr{G}_{\varepsilon^{\prime}}} \rightarrow 0 \quad \text { при } \quad \varepsilon^{\prime} \rightarrow 0 .
$$

Таким образом, в условии С4 предел (3.7) заменяется на (3.11).

Нетрудно проверить, учитывая следствие 3.1, что все утверждения, которые выводятся из условий C1-C4 в [17; гл. III, §1], также будут иметь место и для случая с модифицированным условием С4 для спектральных задач в тонких областях с различными предельными размерностями. Применяя эти утверждения к задачам (3.1) и (3.5), получаем следующие теоремы.

Теорема 2. Пустъ $\left\{\lambda_{n}(\varepsilon)\right\}_{n \in \mathbb{N}} u\left\{\mu_{n}\right\}_{n \in \mathbb{N}}-$ упорядоченные последовательности (3.3) и (3.6) собственных значений задач (3.1) и (3.5) соответственно, $\left\{u_{n}^{\varepsilon}\right\}_{n \in \mathbb{N}}$ - последовательность соответствующих собственных функций задачи (3.1), ортонормированных условием (3.4).

Тогда для каждого $n \in \mathbb{N}$

$$
\lambda_{n}(\varepsilon) \rightarrow \mu_{n} \quad \text { при } \quad \varepsilon \rightarrow 0 .
$$

Кроме того, существует подпоследовательность последовательности $\{\varepsilon\}$, которую вновъ обозначим через $\{\varepsilon\}$, такая, что для каждого $n \in \mathbb{N}$

$$
\mathrm{P}_{\varepsilon} u_{n}^{\varepsilon} \rightarrow v_{n} \quad \text { слабо в } H_{0}^{1}(\Omega) \quad \text { при } \quad \varepsilon \rightarrow 0,
$$

где $\left\{v_{n}\right\}_{n \in \mathbb{N}}-$ собственные функиии задачи (3.5), ортонормированные в $\mathscr{H}_{0}$.

Теорема 3. Для каждого $n \in \mathbb{N}$ при достаточно малых значениях $\varepsilon$

$$
\left|\lambda_{n}(\varepsilon)-\mu_{n}\right| \leqslant c_{1}(n) \sqrt{\varepsilon} .
$$

Предположим, что $\mu_{n}=\mu_{n+1}=\cdots=\mu_{n+q}-q$-кратное собственное значение задачи (3.5). Тогда для любого

$$
v \in N\left(\mu_{n}, A_{0}\right):=\left\{u \in \mathscr{H}_{0}: A_{0} u=\mu_{n} u\right\}, \quad\|v\|_{\mathscr{H}_{0}}=1,
$$


существует линейная комбинация $\widetilde{u}^{\varepsilon}$ собственных векторов $u_{n}^{\varepsilon}, \ldots, u_{n+q}^{\varepsilon}$ задачи (3.1) такая, что

$$
\left\|R_{\varepsilon} v-\widetilde{u}^{\varepsilon}\right\|_{\mathscr{H}_{\varepsilon}} \leqslant c_{2}(n) \sqrt{\varepsilon} .
$$

ЗАмЕЧАНИЕ 3. При выполнении условий следствия 2.1 из (3.9) выводим неравенство

$$
\left\|v-E_{\varepsilon}\left(\widetilde{u}^{\varepsilon}\right)\right\|_{L^{2}(\Omega)} \leqslant c_{3}(n) \sqrt{\varepsilon} .
$$

\section{§4. Асимптотические разложения для собственных значений и собственных функций спектральной задачи Неймана}

В этом параграфе сделаем дополнительные предположения для тонкой области $\Omega_{\varepsilon}$ и коэффициентов дифференциального оператора $L_{\varepsilon}$. Будем считать, что $\Omega=\left\{x^{\prime} \in \mathbb{R}^{n-d}: x_{i} \in(0,1), i=1, \ldots, n-d\right\}$, функции $\left\{h_{ \pm}^{(j)}: j=1, \ldots, d\right\}$ четные по каждой из переменных $\xi_{1}, \ldots, \xi_{n-d}$, ячейка периодичности $\omega_{0}$ симметрична относительно гиперплоскостей $\xi_{i}=1 / 2, i=1, \ldots, n-d$, и $\varepsilon^{-1} \in \mathbb{N}$. Далее, как в п. 1.1, определяем тонкую перфорированную область $\Omega_{\varepsilon}$. Коэффициенты $\left\{a_{i j}\right\}_{i, j=1, \ldots, n}$ оператора $L_{\varepsilon}$ удовлетворяют условиям симметрии

$$
a_{i j}\left(S_{h}^{\prime} \xi\right)=(-1)^{\delta_{i, h}+\delta_{j, h}} a_{i j}(\xi) \quad \forall \xi \in \mathbb{R}^{n}, \quad \forall h=1, \ldots, n-d,
$$

где $S_{h}^{\prime} \xi=\left((-1)^{\delta_{1, h}} \xi_{1}, \ldots,(-1)^{\delta_{n-d, h}} \xi_{n-d}, \xi_{n-d+1}, \ldots, \xi_{n}\right), \delta_{i, h}-$ символ Кронекера.

ЗАмЕчАниЕ 4. Впервые аналогичные условия симметрии появились в монографии [18; гл. $6, \S 3]$. Там было показано, что условия симметрии для краевых задач с быстро осциллирующими коэффициентами позволяют существенно уменьшить объем работы по определению локальных и усредненных характеристик композиционного материала. В работе первого автора [21] аналогичные условия симметрии по всем переменным использовались для упрощения схемы построения полных асимптотических разложений для собственных значений и собственных функций спектральных задач в перфорированном кубе. Кроме того, в [21] был предложен новый вид асимптотического анзаца для собственных функций и было показано, что за счет условий симметрии частичные суммы этих асимптотических рядов не оставляют невязок на внешней границе перфорированного куба, а это, в свою очередь, существенно облегчало обоснование асимптотик.

Для тонких областей условия симметрии модифицируются, а именно они зависят от предельной размерности тонкой области (см. (4.1)) следующим образом: коэффициенты $a_{i i}, i=1, \ldots, n$, четные только по переменным $\xi_{1}, \ldots, \xi_{n-d}$, а функция $a_{i j}(i \neq j)$ четная по переменным $\left\{\xi_{1}, \ldots, \xi_{n-d}\right\} \backslash\left\{\xi_{i}, \xi_{j}\right\}$ и нечетная по переменным $\xi_{i}$ и $\xi_{j}$, если $i, j \in\{1, \ldots, n-d\}$. Примером дифференциального оператора, который удовлетворяет условиям (4.1), является оператор Лапласа.

В $\Omega_{\varepsilon}$ рассмотрим спектральную задачу

$$
\begin{cases}-L_{\varepsilon}\left(u^{\varepsilon}(x)\right)=\lambda(\varepsilon) \rho\left(\frac{x}{\varepsilon}\right) u^{\varepsilon}(x), & x \in \Omega_{\varepsilon}, \\ \sigma_{\varepsilon}\left(u^{\varepsilon}(x)\right)=0, & x \in G_{\varepsilon} \cup S_{\varepsilon}^{ \pm}, \\ u^{\varepsilon}(x)=0, & x \in \Gamma_{\varepsilon} .\end{cases}
$$


Дальше неоднократно будем пользоваться следующим утверждением.

ПредлОЖениЕ 1 (см. [21]). Если функиии $\Phi_{1}, \Phi_{0}^{ \pm}, F_{0}, \partial F_{i} / \partial \xi_{i}, i=1, \ldots, n$ четные (нечетные) по одной из переменных $\xi_{1}, \ldots, \xi_{n-d}$ относительно $1 / 2$, тогда решение задачи (2.10) является четной (нечетной) относительно $1 / 2$ по той же переменной функиией.

Учитывая это утверждение и условия (4.1), легко проверить, что функции $N_{p} \in H_{\sharp}^{1}\left(\omega_{0}\right), p=1, \ldots, n-d$, которые определяются как 1-периодические по $\xi^{\prime}$ решения задач

$$
\left\{\begin{array}{l}
L_{\xi \xi}\left(N_{p}\right)+\partial_{\xi_{i}} a_{i p}=0 \quad \text { в } \quad \omega_{0}, \\
\sigma_{\xi}\left(N_{p}\right)+a_{i p} \nu_{i}=0 \\
\left\langle\rho N_{p}\right\rangle_{\omega_{0}}=0
\end{array}\right.
$$

удовлетворяют соотношениям

$$
N_{p}\left(S_{h}^{\prime} \xi\right)=(-1)^{\delta_{p, h}} N_{p}(\xi), \quad \forall \xi \in \Upsilon, \quad \forall h=1, \ldots, n-d,
$$

где $\Upsilon=\bigcup_{\mathbf{z}_{0} \in \mathbb{Z}^{n}}\left(\bar{\omega}_{0}+\mathbf{z}_{0}\right), \mathbf{z}_{0}=\left(z_{1}, \ldots, z_{n-d}, 0, \ldots, 0\right)$.

На основании (4.4) и (4.1) убеждаемся, что коэффициенты усредненного оператора (см. (2.14)) $\widehat{a}_{p q}=0$ при $p \neq q$. Таким образом, усредненная спектральная задача для задачи (4.2) при таких условиях симметрии будет иметь вид

$$
\begin{cases}-\sum_{p=1}^{n-d} \widehat{a}_{p p} \frac{\partial^{2} u\left(x^{\prime}\right)}{\partial x_{p}^{2}}=\lambda \widehat{\rho} u\left(x^{\prime}\right), & x^{\prime} \in \Omega, \\ u\left(x^{\prime}\right)=0, & x^{\prime} \in \partial \Omega,\end{cases}
$$

где $\widehat{a}_{p p} \equiv\left\langle a_{p p}+\sum_{j=1}^{n} a_{p j} \partial_{\xi_{j}} N_{p}\right\rangle_{\omega_{0}}, p=1, \ldots, n-d$.

Для усредненной задачи (4.5) можно явно определить попарно ортогональные в $L^{2}(\Omega)$ собственные функции

$$
\left\{\prod_{p=1}^{n-d} \sin \left(\pi k_{p} x_{p}\right), x^{\prime} \in \Omega, k_{p} \in \mathbb{N}\right\},
$$

которые формируют плотное множество в пространстве $L^{2}(\Omega)$. Собственной функции $\prod_{p=1}^{n-d} \sin \left(\pi k_{p} x_{p}\right)$ соответствует собственное значение

$$
\lambda=(\widehat{\rho})^{-1}\left(\pi k_{p}\right)^{2} \widehat{a}_{p p}
$$

кратность которого определяется количеством точек с положительными целочисленными координатами, лежащих на поверхности

$$
\lambda=(\widehat{\rho})^{-1} \pi^{2} \sum_{p=1}^{n-d} \widehat{a}_{p p} x_{p}^{2} .
$$

Выпишем все собственные значения задачи (4.5) с учетом их кратности в строKy:

$$
0<\lambda_{1}<\lambda_{2} \leqslant \lambda_{3} \leqslant \cdots \leqslant \lambda_{m} \leqslant \cdots, \quad \lim _{m \rightarrow+\infty} \lambda_{m}=+\infty .
$$


Рассмотрим некоторое собственное значение $\lambda_{m}$ кратности $r$, которое удовлетворяет соотношениям

$$
\lambda_{m-1}<\lambda_{m}=\cdots=\lambda_{m+r-1}=(\widehat{\rho})^{-1} \pi^{2} k_{p, s}^{2} \widehat{a}_{p p}<\lambda_{m+r}, \quad s=1, \ldots, r,
$$

где $\left(k_{1, s}, k_{2, s}, \ldots, k_{n-d, s}\right)$ - точки с целочисленными координатами определяющие $\lambda_{m}$. Через $v_{s}\left(x^{\prime}\right):=\prod_{p=1}^{n-d} \sin \left(\pi k_{p, s} x_{p}\right), x^{\prime} \in \Omega, s=1, \ldots, r$, обозначим собственные функции усредненной задачи (4.5) соответствующие $\lambda_{m}$.

Будем искать асимптотические разложения для собственных функций $u_{m}^{\varepsilon}$, $\ldots, u_{m+r-1}^{\varepsilon}$ и собственных значений $\lambda_{m}(\varepsilon), \ldots, \lambda_{m+r-1}(\varepsilon)$ задачи $(4.2)$ в виде

$$
\begin{aligned}
& R_{\varepsilon}^{(s)}(x):=v_{s}\left(x^{\prime}\right)+\sum_{q=1}^{\infty} \varepsilon^{q} \sum_{\alpha} N_{\alpha}^{(s, q)}\left(\frac{x}{\varepsilon}\right) D^{\alpha} v_{s}\left(x^{\prime}\right), \\
& \Lambda_{\varepsilon}^{(s)}:=\lambda_{m}+\varepsilon^{2} \lambda_{2}^{(s)}+\varepsilon^{4} \lambda_{4}^{(s)}+\cdots, \quad s=1, \ldots, r,
\end{aligned}
$$

где $\alpha=\left(\alpha_{1}, \ldots, \alpha_{n}\right)$ - мультииндекс, $\alpha_{i} \in\{0,1\},|\alpha|=\alpha_{1}+\cdots+\alpha_{n}$,

$$
D^{\alpha} \equiv \frac{\partial^{|\alpha|}}{\partial x_{1}^{\alpha_{1}} \cdots \partial x_{n}^{\alpha_{n}}},
$$

$\left\{N_{\alpha}^{(s, q)}(\xi), \xi \in \omega_{0}\right\}-1$-периодические по $\xi^{\prime}$ функции, которые будут определены ниже.

Подставляя разложения (4.7) и (4.8) при каждом фиксированном $s \in\{1$, $\ldots, r\}$ в уравнения и краевые условия задачи (4.2), разделяя быстрые переменные $\xi=\varepsilon^{-1} x$ и медленные переменные $x$, группируя слагаемые при равных степенях $\varepsilon$, получаем рекуррентную последовательность задач для определения функций $\left\{N_{\alpha}^{(s, q)} \in H_{\sharp}^{1}\left(\omega_{0}\right)\right\}$

$$
\begin{cases}L_{\xi \xi}\left(N_{\alpha}^{(s, q)}\right)+\widetilde{M}_{\alpha}^{(s, q)}(\xi)+\sum_{t=0}^{q-2} \lambda_{t}^{(s)} \rho(\xi) N_{\alpha}^{(s, q-2-t)}(\xi)=0, & \xi \in \omega_{0}, \\ \sigma_{\xi}\left(N_{\alpha}^{(s, q)}\right)+\sum_{i, j=1}^{n} \theta_{\alpha}(j) a_{i j}(\xi) N_{\mu_{j}(\alpha)}^{(s, q-1)}(\xi) \nu_{i}=0, & \xi \in S^{ \pm} \cup \partial T_{0}, \\ \left\langle\rho N_{\alpha}^{(s, q)}\right\rangle_{\omega_{0}}=0, & \end{cases}
$$

где $N_{\alpha}^{(s, 0)}=\delta_{|\alpha|, 0}, N_{\alpha}^{(s, t)} \equiv 0$ при $t<0, \lambda_{0}^{(s)}=\lambda_{m}$ и считаем, что $\lambda_{t}^{(s)}=0$ при нечетном $t$,

$$
\begin{gathered}
\widetilde{M}_{\alpha}^{(s, q)}=\sum_{i, j=1}^{n}\left(\theta_{\alpha}(j) \partial_{\xi_{j}}\left(a_{i j} N_{\mu_{j}(\alpha)}^{(s, q-1)}\right)+\theta_{\alpha}(j) a_{i j} \partial_{\xi_{j}} N_{\mu_{j}(\alpha)}^{(s, q-1)}+\widetilde{\theta}_{\alpha}(i, j) a_{i j} N_{\mu_{i j}(\alpha)}^{(s, q-2)}\right), \\
\mu_{j}(\alpha)=\left(\alpha_{1}, \alpha_{2}, \ldots, \alpha_{j-1}, 1-\alpha_{j}, \alpha_{j+1}, \ldots, \alpha_{n-1}, \alpha_{n}\right), \quad \mu_{i j}(\alpha)=\mu_{i}\left(\mu_{j}(\alpha)\right), \\
\theta_{\alpha}(j)=\left(-\left(\pi k_{j, s}\right)^{2}\right)^{1-\alpha_{j}}, \quad \widetilde{\theta}_{\alpha}(i, j)=\theta_{\alpha}(j) \theta_{\mu_{i}(\alpha)}(j) .
\end{gathered}
$$

Используя условие разрешимости (2.12) для задачи (2.10) и предложение 1 , докажем следующую лемму. 
Лемма 4.1. Рекуррентная последовательность задач (4.9) при каждом фиксированном $s \in\{1, \ldots, r\}$ однозначно разрешима, причем для любого мультииндекса $\alpha$ и любого $q \in \mathbb{N}$

$$
\begin{gathered}
N_{\alpha}^{(s, q)} \equiv 0, \quad \text { если }|\alpha|+q-\text { нечетное число, } \\
N_{\alpha}^{(s, q)}\left(S_{h} \xi\right)=(-1)^{\delta_{\alpha}(h)} N_{\alpha}^{(s, q)}(\xi) \quad \forall \xi \in \Upsilon, \quad \forall h=1, \ldots, n-d,
\end{gathered}
$$

где $\delta_{\alpha}(h):=\delta_{\alpha_{1}, h}+\delta_{2 \alpha_{2}, h}+\cdots+\delta_{(n-d) \alpha_{n-d}, h}$. Кроме того, для любого четного числа $q$

$$
\lambda_{q}^{(s)}=(\widehat{\rho})^{-1} \sum_{i, j=1}^{n}\left\langle\left(\pi k_{i, s}\right)^{2} a_{i j} \frac{\partial}{\partial \xi_{i}}\left(N_{\mu_{j}([0])}^{(s, q+1)}\right)-\widetilde{\theta}_{[0]}(i, j) a_{i j} N_{\mu_{j}([0])}^{(s, q)}\right\rangle_{\omega_{0}},
$$

əде $[0]:=(0, \ldots, 0)$.

ДокАЗАТЕЛЬство. Доказательство проведем методом математической индукции. Соотношения (4.10)-(4.12) при $q=1$ легко проверить, применяя предложение 1 к задачам

$$
\begin{cases}L_{\xi \xi}\left(N_{\alpha}^{(s, 1)}\right)+\theta_{\alpha}(j) \frac{\partial}{\partial \xi_{j}}\left(a_{i j} N_{\mu_{j}(\alpha)}^{(s, 0)}\right)=0, & \xi \in \omega_{0}, \\ \sigma_{\xi}\left(N_{\alpha}^{(s, 1)}\right)+\theta_{\alpha}(j) a_{i j} N_{\mu_{j}(\alpha)}^{(s, 0)} \nu_{i}=0, & \xi \in S^{ \pm} \cup \partial T_{0}, \\ \left\langle\rho N_{\alpha}^{(s, 1)}\right\rangle_{\omega_{0}}=0 . & \end{cases}
$$

Предположим, что для всех $\alpha$ мы уже нашли функции $N_{\alpha}^{(s, q)}, q=1, \ldots, p-1$, удовлетворяющие условиям (4.10) и $(4.11)$, и числа $\lambda_{1}^{(s)}, \lambda_{2}^{(s)}, \ldots, \lambda_{p-3}^{(s)}$.

Тогда, если $p+|\alpha|$ нечетное, то, учитывая предположение индукции (4.10) и соотношения

$$
\begin{gathered}
\left|\mu_{j}(\alpha)\right|=|\alpha|+1-2 a_{j}= \begin{cases}|\alpha|+1, & \alpha_{j}=0, \\
|\alpha|-1, & \alpha_{j}=1,\end{cases} \\
\left|\mu_{i j}(\alpha)\right|= \begin{cases}|\alpha|+2-2 \alpha_{i}-2 \alpha_{j}, & i \neq j, \\
|\alpha|, & i=j,\end{cases}
\end{gathered}
$$

имеем, что

$$
N_{\mu_{j}(\alpha)}^{(s, p-1)} \equiv N_{\mu_{i j}(\alpha)}^{(s, p-2)} \equiv N_{\alpha}^{(s, p-2)} \equiv N_{\alpha}^{(s, p-3-t)} \equiv 0
$$

при четном $t$, а выражение $\sum_{t=0}^{p-3} \lambda_{t}^{(s)} \rho N_{\alpha}^{(s, p-3-t)} \equiv 0$, поскольку при нечетных $t$ по договоренности $\lambda_{t}^{(s)}=0$. Поэтому из (4.9) при $q=p$ получаем, что $N_{\alpha}^{(s, p)} \equiv 0$.

Пусть теперь $p+|\alpha|$ четное. Принимая во внимание условия (4.1) и предположения индукции, выводим следующее соотношение:

$$
\begin{aligned}
& \left.\frac{\partial}{\partial \eta_{i}}\left(a_{i j}(\eta) N_{\mu_{j}(\alpha)}^{(s, p-1)}(\eta)\right)\right|_{\eta=S_{h} \xi}=(-1)^{\delta_{h, i}+\delta_{h, j}+\delta_{h, i}} \frac{\partial a_{i j}(\xi)}{\partial \xi_{i}}(-1)^{\delta_{\mu_{j}(\alpha)}(h)} N_{\mu_{j}(\alpha)}^{(s, p-1)}(\xi) \\
& \quad+(-1)^{\delta_{h, i}+\delta_{h, j}} a_{i j}(\xi)(-1)^{\delta_{\mu_{j}(\alpha)}(h)+\delta_{h, i}} \frac{\partial N_{\mu_{j}(\alpha)}^{(s, p-1)}(\xi)}{\partial \xi_{i}}
\end{aligned}
$$


учитывая то, что

$$
\delta_{\mu_{j}(\alpha)}(h)= \begin{cases}\delta_{\alpha}(h)-\delta_{h, j}, & \text { если } \alpha_{j}=1 \\ \delta_{\alpha}(h)+\delta_{h, j}, & \text { если } \alpha_{j}=0\end{cases}
$$

имеем

$$
\left.\frac{\partial}{\partial \eta_{i}}\left(a_{i j}(\eta) N_{\mu_{j}(\alpha)}^{(s, p-1)}(\eta)\right)\right|_{\eta=S_{h} \xi}=(-1)^{\delta_{\alpha}(h)} \frac{\partial}{\partial \xi_{i}}\left(a_{i j}(\xi) N_{\mu_{j}(\alpha)}^{(s, p-1)}(\xi)\right) \quad \forall \xi \in \Upsilon
$$

и любого $h \in\{1, \ldots, n-d\}$. Аналогично выводим равенства

$$
\begin{gathered}
\left.a_{i j}(\eta) \frac{\partial}{\partial \eta_{i}}\left(N_{\mu_{j}(\alpha)}^{(s, p-1)}(\eta)\right)\right|_{\eta=S_{h} \xi}=(-1)^{\delta_{\alpha}(h)} a_{i j}(\xi) \frac{\partial}{\partial \xi_{i}}\left(N_{\mu_{j}(\alpha)}^{(s, p-1)}(\xi)\right), \\
\left.a_{i j}(\eta) N_{\mu_{i j}(\alpha)}^{(s, p-2)}(\eta)\right|_{\eta=S_{h} \xi}=(-1)^{\delta_{\alpha}(h)} a_{i j}(\xi) N_{\mu_{i j}(\alpha)}^{(s, p-2)}(\xi), \\
\left.\rho(\eta) N_{\alpha}^{(s, p-2)}(\eta)\right|_{\eta=S_{h} \xi}=(-1)^{\delta_{\alpha}(h)} \rho(\xi) N_{\alpha}^{(s, p-2)}(\xi) \quad \forall \xi \in \Upsilon, \quad \forall h=1, \ldots, n-d .
\end{gathered}
$$

Теперь заметим, что если $|\alpha| \neq 0$, то условие разрешимости для задачи (4.9) при $q=p$ автоматически выполнено, поскольку правая часть как в уравнении, так и в краевых условиях задачи (4.9) является нечетной по крайней мере по одной из переменных $\xi_{1}, \ldots, \xi_{n-d}$ функцией и область $\omega_{0}$ симметрична относительно гиперплоскостей $\xi_{i}=1 / 2, i=1, \ldots, n-d$. Если $|\alpha|=0$ и $p$ четное, то, записывая условие разрешимости для задачи (4.9), получим формулу (4.12), которая определяет $\lambda_{p-2}^{(s)}$. Лемма доказана.

4.1. Обоснование построенной асимптотики. Подставляя частичные суммы (индексы $p$ и $s=1, \ldots, r$ фиксированы, $p$ - четное число)

$$
R_{\varepsilon, p}^{(s)}=v_{s}\left(x^{\prime}\right)+\sum_{q=1}^{p} \varepsilon^{q} \sum_{\alpha} N_{\alpha}^{(s, q)}\left(\varepsilon^{-1} x\right) D^{\alpha} v_{s}\left(x^{\prime}\right), \quad \Lambda_{\varepsilon, p}^{(s)}=\lambda_{m}+\sum_{q=1}^{p} \varepsilon^{q} \lambda_{q}^{(s)}
$$

асимптотических разложений (4.7) и (4.8) в уравнения и краевые условия задачи (4.2) и учитывая соотношения (4.9), находим

$$
\begin{gathered}
L_{\varepsilon}\left(R_{\varepsilon, p}^{(s)}\right)+\rho_{\varepsilon} \Lambda_{\varepsilon, p}^{(s)} R_{\varepsilon, p}^{(s)} \\
=\left.\sum_{q=-1}^{p-2} \varepsilon^{q} \sum_{\alpha}\left[L_{\xi \xi}\left(N_{\alpha}^{(s, q+2)}\right)+\widetilde{M}_{\alpha}^{(s, q+2)}+\sum_{l=1}^{q} \rho_{\varepsilon} \lambda_{l}^{(s)} N_{\alpha}^{(s, q-l)}\right]\right|_{\xi=x / \varepsilon} D^{\alpha} v_{s}\left(x^{\prime}\right) \\
+\varepsilon^{p-1} F_{1, \varepsilon}^{(s)}(x)=\varepsilon^{p-1} F_{1, \varepsilon}^{(s)}(x), \quad x \in \Omega_{\varepsilon}, \\
\sigma_{\varepsilon}\left(R_{\varepsilon, p}^{(s)}\right)=\left.\sum_{q=0}^{p-1} \varepsilon^{q} \sum_{\alpha}\left[\left(\sigma_{\xi}\left(N_{\alpha}^{(s, q+1)}\right)+\theta_{\alpha}(j) a_{i j} N_{\mu_{j}(\alpha)}^{(s, q)} \nu_{i}\right)\right]\right|_{\xi=x / \varepsilon} D^{\alpha} v_{s}\left(x^{\prime}\right) \\
+\varepsilon^{p} F_{2, \varepsilon}^{(s)}(x)=\varepsilon^{p} F_{2, \varepsilon}^{(s)}(x), \quad x \in S_{\varepsilon}^{ \pm} \cup G_{\varepsilon},
\end{gathered}
$$

где

$$
\begin{aligned}
\left\|F_{1, \varepsilon}^{(s)}\right\|_{L^{2}\left(\Omega_{\varepsilon}\right)} \leqslant & c_{1}(p) \varepsilon^{d / 2}, \quad\left\|F_{2, \varepsilon}^{(s)}\right\|_{L^{2}\left(S_{\varepsilon}^{ \pm}\right)} \leqslant c_{2}(p) \varepsilon^{d / 2-1 / 2}, \\
& \left\|F_{2, \varepsilon}^{(s)}\right\|_{L^{2}\left(G_{\varepsilon}\right)} \leqslant c_{3}(p) \varepsilon^{d / 2-1 / 2} .
\end{aligned}
$$


Найдем значения $R_{\varepsilon, p}^{(s)}$ на части $\Gamma_{\varepsilon}$ границы области $\Omega_{\varepsilon}$. Для произвольных $\alpha$ и $q \in \mathbb{N}$ рассмотрим $N_{\alpha}^{(s, q)}(x / \varepsilon) D^{\alpha} v_{s}\left(x^{\prime}\right)$. Тогда, если $\alpha_{i}=1$, то в силу $(4.11)$ функция $N_{\alpha}^{(s, q)}$ нечетная, а значит $\left.N_{\alpha}^{(s, q)}\right|_{x_{i}=0}=\left.N_{\alpha}^{(s, q)}\right|_{x_{i}=1}=0$. Если же $\alpha_{i}=0$, то $\left.D^{\alpha} v_{s}\left(x^{\prime}\right)\right|_{x_{i}=0}=\left.D^{\alpha} v_{s}\left(x^{\prime}\right)\right|_{x_{i}=1}=0$. Здесь $i \in\{1, \ldots, n-d\}$.

Таким образом $R_{\varepsilon, p}^{(s)}$ удовлетворяет следующей краевой задаче:

$$
\begin{cases}L_{\varepsilon}\left(R_{\varepsilon, p}^{(s)}\right)+\Lambda_{\varepsilon, p}^{(s)} \rho_{\varepsilon} R_{\varepsilon, p}^{(s)}=\varepsilon^{p-1} F_{1, \varepsilon}^{(s)}, & x \in \Omega_{\varepsilon}, \\ R_{\varepsilon, p}^{(s)}=0, & x \in \Gamma_{\varepsilon}, \\ \sigma_{\varepsilon}\left(R_{\varepsilon, p}^{(s)}\right)=\varepsilon^{p} F_{2, \varepsilon}^{(s)}, & x \in G_{\varepsilon} \cup S_{\varepsilon}^{ \pm},\end{cases}
$$

Домножив задачу (4.15) на произвольную функцию $v \in H^{1}\left(\Omega_{\varepsilon} ; \Gamma_{\varepsilon}\right)$ и проинтегрировав по области $\Omega_{\varepsilon}$, получим

$$
\int_{\Omega_{\varepsilon}} a_{i j}^{\varepsilon} \partial_{x_{i}} R_{\varepsilon, p}^{(s)} \partial_{x_{j}} v d x-\Lambda_{\varepsilon, p}^{(s)}\left(J_{\varepsilon} R_{\varepsilon, p}^{(s)} J_{\varepsilon} v\right)_{\Omega_{\varepsilon}, \rho_{\varepsilon}}=\varepsilon^{p-1} f_{\varepsilon}(v),
$$

где $J_{\varepsilon}: W_{\varepsilon} \mapsto L_{\rho_{\varepsilon}}^{2}\left(\Omega_{\varepsilon}\right)$ - оператор вложения гильбертова пространства $W_{\varepsilon}=$ $\left\{u \in H^{1}\left(\Omega_{\varepsilon} ; \Gamma_{\varepsilon}\right)\right\}$ со скалярным произведением

$$
\langle u, v\rangle_{\varepsilon}=\int_{\Omega_{\varepsilon}} a_{i j}^{\varepsilon} \partial_{x_{i}} u \partial_{x_{j}} v d x
$$

в весовое лебегово пространство (классов) функций $L_{\rho_{\varepsilon}}^{2}\left(\Omega_{\varepsilon}\right)$ со скалярным произведением

$$
(u, v)_{\Omega_{\varepsilon}, \rho_{\varepsilon}}=\int_{\Omega_{\varepsilon}} \rho_{\varepsilon} u v d x
$$

Функционал

$$
\begin{gathered}
f_{\varepsilon}^{(s)}(v)=-\int_{\Omega_{\varepsilon}} F_{\varepsilon, 1}^{(s)}(x) v(x) d x+\varepsilon \int_{S_{\varepsilon}^{ \pm}} F_{\varepsilon, 2}^{(s)}(x) v(x) d \sigma_{x}+\varepsilon \int_{G_{\varepsilon}} F_{\varepsilon, 2}^{(s)}(x) v(x) d \sigma_{x}, \\
v \in W_{\varepsilon},
\end{gathered}
$$

является линейным и ограниченным в $W_{\varepsilon}$, причем ввиду $(2.6),(2.7)$ и $(4.14)$ $\left\|f_{\varepsilon}^{(s)}\right\| \leqslant c \varepsilon^{d / 2}$. Следовательно, на основании теоремы Рисса

$$
\forall s=1, \ldots, r \quad \exists ! \varphi_{\varepsilon}^{(s)} \in W_{\varepsilon}: f_{\varepsilon}^{(s)}(v)=\left\langle\varphi_{\varepsilon}^{(s)}, v\right\rangle_{\varepsilon} \quad \forall v \in W_{\varepsilon}
$$

и $\left\|f_{\varepsilon}^{(s)}\right\|=\left\|\varphi_{\varepsilon}^{(s)}\right\|_{W_{\varepsilon}}$. Определим оператор $A_{\varepsilon} \equiv J_{\varepsilon}^{*} J_{\varepsilon}: W_{\varepsilon} \mapsto W_{\varepsilon}$. Легко проверить (см., например, [21]), что этот оператор является самосопряженным, положительным и компактным, а исходная спектральная задача (4.2) эквивалентна спектральной задаче для оператора $A_{\varepsilon}$ :

$$
A_{\varepsilon} u^{\varepsilon}=\lambda^{-1}(\varepsilon) u^{\varepsilon}, \quad u_{\varepsilon} \in W_{\varepsilon} .
$$

Перепишем теперь равенство (4.16) в новых обозначениях:

$$
\left\langle R_{\varepsilon, p}^{(s)}, v\right\rangle_{\varepsilon}-\Lambda_{\varepsilon, p}^{(s)}\left\langle A_{\varepsilon}\left(R_{\varepsilon, p}^{(s)}\right), v\right\rangle_{\varepsilon}=\varepsilon^{p-1}\left\langle\varphi_{\varepsilon}^{(s)}, v\right\rangle_{\varepsilon} \quad \forall v \in W_{\varepsilon},
$$


откуда получим операторное равенство

$$
R_{\varepsilon, p}^{(s)}-\Lambda_{\varepsilon, p}^{(s)} A_{\varepsilon}\left(R_{\varepsilon, p}^{(s)}\right)=\varepsilon^{p-1} \varphi_{\varepsilon}^{(s)} .
$$

Так как $\left\|\varphi_{\varepsilon}^{(s)}\right\|_{W_{\varepsilon}}=\left\|f_{\varepsilon}^{(s)}\right\| \leqslant c \varepsilon^{d / 2}$ и при достаточно малых $\varepsilon$

$$
0<\widetilde{c}_{1} \leqslant \Lambda_{\varepsilon, p}^{(s)} \leqslant \widetilde{c}_{2}, \quad 0<\widetilde{c}_{3} \leqslant\left\|R_{\varepsilon, p}^{(s)}\right\|_{W_{\varepsilon}} \leqslant \widetilde{c}_{4},
$$

то из (4.17) следует неравенство

$$
\left\|A_{\varepsilon}\left(\omega_{\varepsilon, p}^{(s)}\right)-\left(\Lambda_{\varepsilon, p}^{(s)}\right)^{-1} \omega_{\varepsilon, p}^{(s)}\right\|_{W_{\varepsilon}} \leqslant \beta_{p}(\varepsilon)
$$

где $\omega_{\varepsilon, p}^{(s)}=R_{\varepsilon, p}^{(s)} \cdot\left\|R_{\varepsilon, p}^{(s)}\right\|_{W_{\varepsilon}}^{-1}, \beta_{p}(\varepsilon)=C \varepsilon^{p+d / 2-1}$. В дальнейшем мы неоднократно будем применять к неравенству (4.19) следующую лемму.

ЛЕмма 4.2 (см. [8]). Пусть $A: H \mapsto H$ - самосопряженный, положительный и компактный оператор. Предположим, что существуют элемент $u \in H,\|u\|_{H}=1, u \mu \in \mathbb{R}$ такие, что $\|A u-\mu u\|_{H} \leqslant \beta$.

Тогда:

1) существует такое собственное значение $\lambda$ оператора $A$, что $|\mu-\lambda| \leqslant \beta$;

2) для всех $d_{0}>\beta$ существует $\widetilde{u} \in H,\|\widetilde{u}\|_{H}=1$ такое, что

$$
\|u-\widetilde{u}\|_{H} \leqslant 2 d_{0}^{-1} \beta,
$$

где $\widetilde{u}$ - линейная комбинация собственных функций оператора $A$, которая соответствует собственным значениям из интервала $\left(\mu-d_{0}\right.$, $\left.\mu+d_{0}\right)$.

Предположим, что построенные асимптотические ряды $\Lambda_{\varepsilon}^{(s)}, s=1, \ldots, r$, распадаются на $k$ серий, т.е. существует четное число $l$ такое, что для любого $p \geqslant l$ при достаточно малых $\varepsilon$

$$
0<\Lambda_{\varepsilon, p}^{\left(\tau_{1,1}\right)}=\cdots=\Lambda_{\varepsilon, p}^{\left(\tau_{1, r_{1}}\right)}<\cdots<\Lambda_{\varepsilon, p}^{\left(\tau_{k, 1}\right)}=\cdots=\Lambda_{\varepsilon, p}^{\left(\tau_{k, r_{k}}\right)},
$$

$r_{0}=0, r_{1}+\cdots+r_{k}=r, \tau_{i, t}=r_{1}+\cdots+r_{i-1}+t$.

Дальше, как в [21; теорема 3.1], доказываем следующую теорему.

Теорема 4. Пусть $\lambda_{m}-r$-кратное собственное значение усредненной задачи $(4.5) ; R_{\varepsilon}^{(s)}, \Lambda_{\varepsilon}^{(s)}$ - соответствующие асимптотические ряды (4.7) и (4.8), причем $\Lambda_{\varepsilon}^{(s)}, s=1, \ldots, r$, распадаются на $k$ серий (4.20).

Тогда для любого четного $p \in \mathbb{N}$ и любых $i \in\{1, \ldots, k\}, t \in\left\{1, \ldots, r_{i}\right\}$ при достаточно мальх значениях $\varepsilon$

$$
\begin{gathered}
\left|\lambda_{m+\tau_{i, t}-1}(\varepsilon)-\Lambda_{\varepsilon, p}^{\left(\tau_{i, 1}\right)}\right| \leqslant C_{1} \varepsilon^{p+2}, \\
\left\|\widetilde{u}_{\varepsilon}-R_{\varepsilon, p}^{\left(\tau_{i, t}\right)}\right\|_{H^{1}\left(\Omega_{\varepsilon}\right)} \leqslant C_{2} \varepsilon^{p+d / 2},
\end{gathered}
$$

где функция $\widetilde{u}_{\varepsilon}-$ линейная комбинация собственных функиий $u_{m+r_{1}+\cdots+r_{i-1}}^{\varepsilon}$, $\ldots, u_{m+r_{1}+\cdots+r_{i}-1}^{\varepsilon}$ задачи (4.2), которые ортонормированы условиями (3.4).

Если для некоторой серии $r_{i}=1$, то определена постоянная $d_{\varepsilon, p}$ такая, что

$$
\left\|d_{\varepsilon, p} u_{m+r_{1}+\cdots+r_{i}-1}^{\varepsilon}-R_{\varepsilon, p}^{\left(\tau_{i, 1}\right)}\right\|_{H^{1}\left(\Omega_{\varepsilon}\right)} \leqslant C_{3} \varepsilon^{p+d / 2} .
$$


ДокАЗАТЕльство. В силу (4.20) имеем, что для любых $i, j \in\{1, \ldots, k\}$, $i \neq j$ при достаточно малых $\varepsilon$

$$
\left|\left(\Lambda_{\varepsilon, p}^{\left(\tau_{i, 1}\right)}\right)^{-1}-\left(\Lambda_{\varepsilon, p}^{\left(\tau_{j, 1}\right)}\right)^{-1}\right| \geqslant c \varepsilon^{l} .
$$

Рассмотрим интервалы

$$
\begin{gathered}
I_{0}=\left\{\mu \in \mathbb{R}:\left|\mu-\left(\lambda_{m}\right)^{-1}\right|<\delta\right\}, \\
I_{p}^{(i)}(\varepsilon)=\left\{\mu \in \mathbb{R}:\left|\mu-\left(\Lambda_{\varepsilon, p}^{\left(\tau_{i, 1}\right)}\right)^{-1}\right|<\beta_{p}(\varepsilon)\right\}, \quad i=1, \ldots, k,
\end{gathered}
$$

где $\delta=1 / 4 \min \left\{\left(\lambda_{m-1}\right)^{-1}-\left(\lambda_{m}\right)^{-1} ;\left(\lambda_{m}\right)^{-1}-\left(\lambda_{m+r}\right)^{-1}\right\}$. Тогда на основании теоремы $3,(4.19),(4.24)$ и первого утверждения леммы 4.2 существует такое $\varepsilon_{0}$, что для любых значений $\varepsilon \in\left(0, \varepsilon_{0}\right)$ верны следующие утверждения:

(H1) в интервале $I_{0}$ содержатся только собственные значения $\left(\lambda_{m+r-1}(\varepsilon)\right)^{-1} \leqslant$ $\cdots \leqslant\left(\lambda_{m}(\varepsilon)\right)^{-1}$ оператора $A_{\varepsilon}$

(Н2) $I_{p+4 d}^{(i)}(\varepsilon) \subset I_{p}^{(i)}(\varepsilon) \subset I_{0}, i=1, \ldots, k, I_{p}^{(i)}(\varepsilon) \cap I_{p}^{(j)}(\varepsilon)=\varnothing, i \neq j$, причем интервал $I_{p}^{(i)}$ находится на числовой прямой правее интервала $I_{p}^{(i+1)}(\varepsilon)$, $i=1, \ldots, r-1$;

(Н3) интервалы $I_{p}^{(i)}(\varepsilon), i=1, \ldots, k$, содержат собственные значения оператоpa $A_{\varepsilon}$.

Покажем, что для каждого $i \in\{1, \ldots, k\}$ при достаточно малых $\varepsilon$ справедливы включения

$$
\left(\lambda_{m+\tau_{i, t}-1}(\varepsilon)\right)^{-1} \in I_{p}^{(i)}(\varepsilon), \quad t=1, \ldots, r_{i},
$$

из которых будут следовать неравенства (4.21).

Для этого, учитывая (Н1) и (Н2), достаточно показать, что каждый интервал $I_{p}^{(i)}(\varepsilon)$ содержит ровно $r_{i}$ собственных значений оператора $A_{\varepsilon}$.

Пусть $r_{1}>1$. В случае, если все $r_{i}=1$, включения (4.25) непосредственно следуют из (Н1)-(Н3). Предположим, что количество собственных значений оператора $A_{\varepsilon}$, которые попадают в интервал $I_{p}^{(1)}(\varepsilon)$ при достаточно малых $\varepsilon$ равно $r_{1}^{\prime}$ и $r_{1}^{\prime}<r_{1}$. Учитывая утверждение $(\mathrm{H} 2)$, в лемме 4.2, примененной к (4.19) при $s=1, \ldots, r_{1}$, можно выбрать постоянную $d_{0}$ так, чтобы

$$
d_{0}>\beta_{p+4 d}(\varepsilon), \quad \varepsilon^{p+d / 2+4 d-1} d_{0}^{-1}=O\left(\varepsilon^{d / 2+1 / 2}\right) \quad \text { при } \varepsilon \rightarrow 0,
$$

и в интервале $\left\{\mu:\left|\mu-\left(\Lambda_{\varepsilon, p+4 d}^{(1)}\right)^{-1}\right|<d_{0}\right\}$ количество собственных значений оператора $A_{\varepsilon}$ вновь было меньше чем $r_{1}$. Тогда из второго утверждения леммы 4.2 следует, что

$$
\left\|\omega_{\varepsilon, p+4 d}^{(s)}-\widetilde{u}_{\varepsilon}\right\|_{W_{\varepsilon}} \leqslant C \varepsilon^{d / 2+1 / 2}, \quad s=1, \ldots, r_{1},
$$

где

$$
\widetilde{u}_{\varepsilon}=\sum_{t=1}^{r_{1}^{\prime}} \alpha_{s t}(\varepsilon) u_{m+t-1}^{\varepsilon}, \quad \sum_{t=1}^{r_{1}^{\prime}}\left(\alpha_{s t}(\varepsilon)\right)^{2} \lambda_{m+t-1}(\varepsilon)=1 .
$$

Благодаря (4.18) и оценке $\|u\|_{H^{1}\left(\Omega_{\varepsilon}\right)} \leqslant c_{1}\|u\|_{W_{\varepsilon}}, u \in W_{\varepsilon}$, из (4.26) получаем, что

$$
\left\|R_{\varepsilon, p+4 d}^{(s)}-\sum_{t=1}^{r_{1}^{\prime}} \widetilde{\alpha}_{s t}(\varepsilon) u_{m+t-1}^{\varepsilon}\right\|_{H^{1}\left(\Omega_{\varepsilon}\right)} \leqslant c_{2} \varepsilon^{d / 2+1 / 2}, \quad s=1, \ldots, r_{1},
$$


где $\widetilde{\alpha}_{s t}(\varepsilon)=\alpha_{s t}(\varepsilon)\left\|R_{\varepsilon, p+4 d}^{(s)}\right\|_{W_{\varepsilon}}, 0<\widetilde{c}_{1} \leqslant \sum_{t=1}^{r_{1}^{\prime}}\left(\widetilde{\alpha}_{s t}(\varepsilon)\right)^{2} \leqslant \widetilde{c}_{2}$. Используя неравенство (3.10), из (4.27) выводим неравенство

$$
\left\|v_{s}-\sum_{t=1}^{r_{1}^{\prime}} \widetilde{\alpha}_{s t}(\varepsilon) \mathrm{P}_{\varepsilon} u_{m+t-1}^{\varepsilon}\right\|_{L^{2}(\Omega)} \leqslant c_{3} \varepsilon^{1 / 2}, \quad s=1, \ldots, r_{1} .
$$

Принимая во внимание теорему 2 и переходя к пределу в (4.28) по некоторой подпоследовательности $\varepsilon^{\prime}$ последовательности $\varepsilon$, имеем

$$
\left\|v_{s}-\sum_{t=1}^{r_{1}^{\prime}} \widehat{\alpha}_{s t} u_{m+t-1}\right\|_{L_{2}(\Omega)}=0, \quad s=1, \ldots, r_{1} \quad\left(r_{1}^{\prime}<r_{1}\right),
$$

где $0<\widetilde{c}_{3} \leqslant \sum_{t=1}^{r_{1}^{\prime}} \widehat{\alpha}_{s t} \leqslant \widetilde{c}_{4}$. Получили противоречие: $r_{1}$ линейно независимых функций выражаются через линейную комбинацию линейно независимых функций, количество которых меньше $r_{1}$.

В интервал $I_{p}^{(1)}(\varepsilon)$ при достаточно малых $\varepsilon$ не может попадать больше чем $r_{1}$ собственных значений оператора $A_{\varepsilon}$, так как в каком-то из интервалов $I_{p}^{(2)}(\varepsilon)$, $\ldots, I_{p}^{(k)}(\varepsilon)$, например $I_{p}^{(2)}(\varepsilon)$, окажется меньше чем $r_{2}$ собственных значений оператора $A_{\varepsilon}$. А этого, как показано выше, не может быть.

Итак, имеют место включения (4.25). Далее, в силу (3.12) и (4.18) из (4.25) получаем оценки

$$
\left|\lambda_{m+\tau_{i, t}-1}(\varepsilon)-\Lambda_{\varepsilon, p}^{\left(\tau_{i, t}\right)}\right| \leqslant c_{1} \varepsilon^{p+d / 2-1}, \quad i=1, \ldots, k, \quad t=1, \ldots, r_{i},
$$

откуда выводим

$$
\left|\lambda_{m+\tau_{i, t}-1}(\varepsilon)-\left(\lambda_{m}+\varepsilon^{2} \lambda_{2}^{\left(\tau_{i, t}\right)}+\varepsilon^{4} \lambda_{4}^{\left(\tau_{i, t}\right)}+\cdots+\varepsilon^{p-4} \lambda_{p-4}^{\left(\tau_{i, t}\right)}\right)\right| \leqslant c_{2} \varepsilon^{p-2},
$$

для всех $i \in\{1, \ldots, k\}$ и $t \in\left\{1, \ldots, r_{i}\right\}$. Таким образом, асимптотические оценки (4.21) доказаны.

Докажем оценки (4.22). Для этого применим вторую часть леммы 4.2 к неравенствам (4.19), выбрав в силу (4.24) постоянную $d_{0}$ так, чтобы $d_{0}=c \varepsilon^{l}$, $d_{0}>\beta_{p}(\varepsilon)$ и интервалы

$$
\left\{\mu \in \mathbb{R}:\left|\mu-\left(\Lambda_{\varepsilon, p}^{\left(\tau_{i, 1}\right)}\right)^{-1}\right|<d_{0}\right\}, \quad i=1, \ldots, k,
$$

не пересекались. Тогда, учитывая (4.25), имеем, что для любых $i=1, \ldots, k$, $t=1, \ldots, r_{i}$ при достаточно малом $\varepsilon$

$$
\left\|\omega_{\varepsilon, p}^{\left(\tau_{i, t}\right)}-\widehat{u}_{\varepsilon}\right\|_{W_{\varepsilon}} \leqslant c_{4} \varepsilon^{p-1-l+d / 2}, \quad\|\widehat{u}\|_{W_{\varepsilon}}=1,
$$

где $\widehat{u}_{\varepsilon}-$ линейная комбинация собственных функций $u_{m+\tau_{i, 1}-1}^{\varepsilon}, \ldots, u_{m+\tau_{i, r_{i}}-1}^{\varepsilon}$. Согласно (4.18) из (4.29) получаем (4.22).

Пусть, например, $r_{1}=1$. Тогда из (4.29) имеем

$$
\left\|\frac{R_{\varepsilon, p}^{(1)}}{\left\|R_{\varepsilon, p}^{(1)}\right\|_{W_{\varepsilon}}}-\frac{u_{m}^{\varepsilon}}{\sqrt{\lambda_{m}(\varepsilon)}}\right\|_{W_{\varepsilon}} \leqslant c_{5} \varepsilon^{p-l-1+d / 2} .
$$


Так как при достаточно малых $\varepsilon$

$$
\begin{gathered}
\left|\left(\lambda_{m}(\varepsilon)\right)^{-1 / 2}-\left(\Lambda_{\varepsilon, p-l-2+2 d}^{(1)}\right)^{-1 / 2}\right| \leqslant c_{6} \varepsilon^{p-l+2 d} \leqslant c_{7} \varepsilon^{p-l-1+d / 2}, \\
\left\|R_{\varepsilon, p}^{(1)}-R_{\varepsilon, p-l-1}^{(1)}\right\|_{W_{\varepsilon}} \leqslant c_{8} \varepsilon^{p-l-1+d / 2},
\end{gathered}
$$

то из $(4.30),(4.18)$ и (4.21) вытекает, что

$$
\left\|\frac{R_{\varepsilon, p-l-1}^{(1)}}{\left\|R_{\varepsilon, p-l-1}^{(1)}\right\|_{W_{\varepsilon}}}-\frac{u_{m}^{\varepsilon}}{\left(\Lambda_{\varepsilon, p-l-2+2 d}^{(1)}\right)^{1 / 2}}\right\|_{W_{\varepsilon}} \leqslant c \varepsilon^{p-l-1+d / 2},
$$

откуда получаем $(4.23)$ с $d_{\varepsilon, p}=\left\|R_{\varepsilon, p}^{\left(\tau_{i, 1}\right)}\right\|_{W_{\varepsilon}} /\left(\Lambda_{\varepsilon, p-1+2 d}^{(1)}\right)^{1 / 2}$. Таким образом, теорема доказана.

Из асимптотических неравенств (4.21) следует такое утверждение.

СлЕДСТвиЕ 4.1. Для каждого $m \in \mathbb{N}$ при достаточно малых $\varepsilon$

$$
\left|\lambda_{m}(\varepsilon)-\lambda_{m}\right| \leqslant c(m) \varepsilon^{2}
$$

где $\lambda_{m}(\varepsilon)$ - m-е собственное значение задачи (4.2), а $\lambda_{m}$ - собственное значение усредненной задачи (4.5).

Асимптотические оценки (4.31) для скорости сходимости собственных значений существенно лучше, чем аналогичные оценки (3.12) из теоремы 3.

ЗАмечАниЕ 5. Аналогичные результаты для задачи (4.2) получаются, если на частях границы $\Gamma_{\varepsilon}$ перфорированной области $\Omega_{\varepsilon}$ заданы или условия Неймана, или периодические условия.

\section{Список литературы}

[1] А. Л. Гольденвейзер, "Построение приближенной теории изгиба пластинки методом асимптотического интегрирования уравнений теории упругости", ПММ, 26:4 (1962), 668-686; англ. пер.: A. L. Gol'denveizer, "Derivation of an approximate theory of bending of a plate by the method of asymptotic integration of the equations of the theory of elasticity", J. Appl. Math. Mech., 26:4 (1962), 1000-1025.

[2] А. Л. Гольденвейзер, Теория упругих тонких оболочек, 2-е изд., Наука, М., 1976; англ. пер. 1-го изд.: А.L. Gol'denveizer, Theory of elastic thin shells, Pergamon Press, Oxford-London-New York-Paris, 1961.

[3] М. Г. Джавадов, “Асимптотика решения краевой задачи для эллиптических уравнений второго порядка в тонких областях", Дифферени. уравнения, 4:10 (1968), 1901-1909.

[4] С. А. Назаров, “Структура решений краевой задачи для эллиптических уравнений в тонких областях", Вестн. ЛГУ. Сер. матем., мех., астрон., 1982, №2, 65-68.

[5] С. Н. Леора, С. А. Назаров , А. В. Проскура, "Вывод предельных уравнений для эллиптических задач в тонких областях при помощи ЭВМ", ЖК. вычисл. матем. и матем. физ., $26: 7$ (1986), 1032-1048; англ. пер.: S. N. Leora, S. A. Nazarov, A. V. Proskura, "Derivation of limiting equations for elliptic problems in thin domains using computers", U.S.S.R. Comput. Math. Math. Phys., 26:4 (1986), 47-58. 
[6] А.Б. Васильева, В.Ф. Бутузов, Асимптотические методы в теории сингулярных возмущений, Высшая школа, М., 1990.

[7] D. Caillerie, "Thin elastic and periodic plates", Math. Methods Appl. Sci., 6:2 (1984), 159-191.

[8] М.И. Вишик, Л.А. Люстерник, "Регулярное вырождение и пограничный слой для линейных дифференциальных уравнений с малым параметром", УМH, 12:5 (1957), 3-122.

[9] Г. П. Панасенко, М. В. Резцов, “Осреднение трехмерной задачи теории упругости в неоднородной пластине”, Докл. АН СССР, 294:5 (1987), 1061-1065; англ. пер.: G. P. Panasenko, M. V. Reztsov, "Averaging a three-dimensional problem of elasticity theory in an inhomogeneous plate", Soviet Math. Dokl., 35:3 (1987), 630-634.

[10] Т. А. Мельник, "Осереднение эллиптических уравнений, описывающих процессы в сильно неоднородных тонких перфорованных областях с быстро изменяющейся толщиной”, Докл. АН УССР, 10 (1991), 15-18.

[11] R. V. Korn, M. Vogelius, "A new model for thin plates with rapidly varying thickness. II. A convergence proof", Quart. Appl. Math., 43:1 (1985), 1-22.

[12] А.Г. Колпаков, "Определяющие уравнения тонкой упругой напряженной балки периодической структуры", ПММ, 63:3 (1999), 513-523; англ. пер.: A. G. Kolpakov, "The governing equations of a thin elastic stressed beam with a periodic structure", J. Appl. Math. Mech., 63:3 (1999), 495-504.

[13] G. A. Chechkin, E. A. Pichugina, "Weighted Korn's inequality for a thin plate with a rough surface", Russ. J. Math. Phys., 7:3 (2000), 279-287.

[14] Т. А. Мельник, А. В. Попов, “Асимптотические аппроксимации решений параболических краевых задач в тонких перфорированных областях с быстро изменяющейся толщиной”, Проблемы матем. анализа, 42 (2009), 43-64; англ. пер.: T. A. Mel'nik, A.V. Popov, "Asymptotic approximations of solutions to parabolic boundary value problems in thin perforated domains of rapidly varying thickness", $J$. Math. Sci. (N. Y.), 162:3 (2009), 348-372.

[15] С.А. Назаров, "Общая схема осреднения самосопряженных эллиптических систем в многомерных областях, в том числе тонких", Алгебра и анализ, 7:5 (1995), 1-92; англ. пер.: S. A. Nazarov, "General averaging procedure for selfadjoint elliptic systems in many-dimensional domains, including thin ones", St. Petersburg Math. J., 7:5 (1996), 681-748.

[16] С. А. Назаров, Асимптотическая теория тонких пластин и стержней. Понижение размерности и интегралъные оценки, Научная книга, Новосибирск, 2002.

[17] О.А. Олейник, Г.А. Иосифьян, А. С. Шамаев, Математические задачи теории силъно неоднородных упругих сред, Изд-во МГУ, М., 1990; англ. пер.: O. A. Ole'inik, A.S. Shamaev, G. A. Yosifyan, Mathematical problems in elasticity and homogenization, Stud. Math. Appl., 26, North-Holland, Amsterdam, 1992.

[18] Н. С. Бахвалов, Г. П. Панасенко, Осреднение процессов в периодических средах, Наука, M., 1984; англ. пер.: N.S. Bakhvalov, G. Panasenko, Homogenisation: Averaging processes in periodic media, Math. Appl. (Soviet Ser.), 36, Kluwer Acad. Publ., Dordrecht, 1989.

[19] Д. Гилбарг, Н. С. Трудингер, Эллиптические дифференииалъные уравнения с частными производными второго порядка, Наука, М., 1989; пер. с англ.: D. Gilbarg, N.S. Trudinger, Elliptic partial differential equations of second order, Grundlehren Math. Wiss., 224, Springer-Verlag, Berlin, 1983.

[20] О.А. Ладыженская, Краевые задачи математической физики, Наука, М., 1973; англ. пер.: O.A. Ladyzhenskaya, The boundary value problems of mathematical physics, Appl. Math. Sci., 49, Springer-Verlag, New York, 1985. 
[21] Т. А. Мельник, “Асимптотические разложения собственных значений и собственных функций эллиптических краевых задач с быстроосциллирующими коэффициентами в перфорированном кубе", Тр. сем. им. И. Г. Петровского, 17, Изд-во Моск. ун-та, М., 1994, 51-88; англ. пер.: T. A. Mel'nik, "Asymptotic expansions of eigenvalues and eigenfunctions for elliptic boundary-value problems with rapidly oscillating coefficients in a perforated cube", J. Math. Sci., 75:3 (1995), 1646-1671.

Т. А. Мельник (T. А. Mel'nik)

Киевский национальный университет

им. Т. Г. Шевченко, Украина

E-mail: melnyk@imath.kiev.ua

\section{А. В. Попов (А. V. Popov)}

Киевский национальный университет

им. Т. Г. Шевченко, Украина

E-mail: popov24@mail.univ.kiev.ua
Поступила в редакцию 18.03.2011 и 27.12 .2011 